\title{
NON-SEMISIMPLE QUANTUM INVARIANTS AND TQFTS FROM SMALL AND UNROLLED QUANTUM GROUPS
}

\author{
MARCO DE RENZI, NATHAN GEER, AND BERTRAND PATUREAU-MIRAND
}

\begin{abstract}
We show that unrolled quantum groups at odd roots of unity give rise to relative modular categories. These are the main building blocks for the construction of $1+1+1$-TQFTs extending CGP invariants, which are non-semisimple quantum invariants of closed 3-manifolds decorated with ribbon graphs and cohomology classes. When we consider the zero cohomology class, these quantum invariants are shown to coincide with the renormalized Hennings invariants coming from the corresponding small quantum groups.
\end{abstract}

The goal of this paper is two-fold: first of all, we provide a new family of concrete examples of relative modular categories. These are ribbon categories which can be used as fundamental bricks for the construction of non-semisimple quantum invariants of closed manifolds in dimension 3 [5] and Extended Topological Quantum Field Theories (ETQFTs) in dimension $1+1+1$ [8]. They are modeled on categories of finite-dimensional weight representations of the unrolled quantum group $U_{q}^{H} \mathfrak{s l}_{2}$ at even roots of unity $q$, which were used in [2] to build TQFTs in dimension $2+1$. They should be thought of as a non-semisimple analogue to standard modular categories, although differences with respect to their semisimple counterparts are many: first of all, a relative modular category $\mathscr{C}$ comes equipped with a structure group $G$ that provides a grading on its objects; secondly, it enjoys finiteness properties only up to the action of a periodicity group $Z$ of transparent objects; more importantly, it is only generically semisimple, with non-semisimple part confined to a critical set $X \subset G$ whose complement is dense in $G$. When $G=Z=\{0\}$ and $X=\varnothing$ the definition reduces to the standard one of [32], see Section 1.7 of [8]. In Theorem 1.3 we prove that categories $\mathscr{C}^{H}$ of finite-dimensional weight representations of unrolled quantum groups $U_{q}^{H} \mathfrak{g}$ associated with arbitrary simple complex Lie algebras $\mathfrak{g}$ at odd roots of unity $q$ are relative modular. These categories were already known to induce topological invariants $\mathrm{N}_{\mathscr{C} H}$ of certain decorated closed 3-manifolds $(M, T, \omega)$, where $T \subset M$ is a $\mathscr{C}^{H}$-colored ribbon graph, where $\omega \in H^{1}(M \backslash T ; G)$ is a cohomology class, and where the triple $(M, T, \omega)$ is subject to a crucial $a d$ missibility condition. These so-called Costantino-Geer-Patureau (CGP) quantum invariants are quite rich, but their definition involves certain technical aspects, such as the notion of computable surgery presentation introduced in [5]. The results of this paper imply these invariants can be extended to graded $1+1+1$-TQFTs for all simple complex Lie algebras $\mathfrak{g}$. In the case of $\mathfrak{s l}_{2}$, these invariants contain the Akutsu-Deguchi-Ohtsuki invariants of colored links and the abelian Reidemeister torsion of closed 3-manifolds, and they were already known to extend to graded $1+1+1$-TQFTs.

The second main result of this paper is a Hennings-type formula for these CGP quantum invariants. More precisely, every simple complex Lie algebra $\mathfrak{g}$ also determines a corresponding small quantum group $\bar{U}_{q} \mathfrak{g}$ for every odd root of unity $q$ $[26,27]$. These finite-dimensional factorizable quotients have been studied a lot in literature [28, 24, 25], and they induce renormalized Hennings TQFTs in dimension $2+1$, see [9]. In particular, their categories of finite-dimensional representations $\overline{\mathscr{C}}$ yield quantum invariants $\mathrm{H}_{\overline{\mathscr{C}}}^{\prime}$ of certain admissible closed 3 -manifolds $(M, T)$, 
where $T \subset M$ is a $\overline{\mathscr{C}}$-colored bichrome graph, which is a very mild generalization of standard ribbon graphs obtained by specifying special components which correspond to surgery presentations. Then, we prove in Theorem 1.4 that, for a fixed simple complex Lie algebra $\mathfrak{g}$ at an odd root of unity $q$, the CGP invariant $\mathrm{N}_{\mathscr{C} H}$ of decorated closed 3-manifolds with zero cohomology classes coincides with the corresponding renormalized Hennings invariant $\mathrm{H}_{\overline{\mathscr{C}}}^{\prime}$. This result builds a bridge between the two theories, and, in this setting, it gives us a way of computing CGP quantum invariants which bypasses computable surgery presentations.

Acknowledgments. We would like to thank the referee for their extremely careful review of our paper. Their deep understanding of our results and their detailed comments helped us improve the paper. NG was partially supported by NSF grants DMS-1308196 and DMS-1452093.

\section{OVERVIEW OF NON-SEMISIMPLE CONSTRUCTIONS}

In this section, we quickly review the two main constructions this paper deals with. References are provided by [5, 2, 8] for the CGP theory, and by [9] for the renormalized Hennings one. All the manifolds we consider are always assumed to be oriented.

1.1. Ribbon categories and m-traces. First, let us fix our notation and conventions for categorical structures. Following [11], a ribbon category is a braided rigid monoidal category $\mathscr{C}$ equipped with a natural transformation $\vartheta: \operatorname{id}_{\mathscr{C}} \Rightarrow \operatorname{id}_{\mathscr{C}}$, called the twist, satisfying

$$
\vartheta_{V \otimes V^{\prime}}=\left(\vartheta_{V} \otimes \vartheta_{V^{\prime}}\right) \circ c_{V^{\prime}, V} \circ c_{V, V^{\prime}}, \quad\left(\vartheta_{V}\right)^{*}=\vartheta_{V^{*}}
$$

for all $V, V^{\prime} \in \mathscr{C}$, where $c_{V, V^{\prime}}: V \otimes V^{\prime} \rightarrow V^{\prime} \otimes V$ denotes the braiding of $\mathscr{C}$. For every $V \in \mathscr{C}$ we denote with

$$
\begin{array}{ll}
\overleftarrow{\mathrm{ev}}_{V}: V^{*} \otimes V \rightarrow \mathbb{1}, & \overleftarrow{\operatorname{coev}}_{V}: \mathbb{1} \rightarrow V \otimes V^{*}, \\
\overrightarrow{\mathrm{ev}}_{V}: V \otimes V^{*} \rightarrow \mathbb{1}, & \overrightarrow{\operatorname{coev}}_{V}: \mathbb{1} \rightarrow V^{*} \otimes V
\end{array}
$$

its left and right duality morphisms, and for every $f \in \operatorname{End}_{\mathscr{C}}(V)$ we denote with $\operatorname{tr}_{\mathscr{C}}(f)$ its categorical trace. As shown in [32], every ribbon category $\mathscr{C}$ induces a ribbon functor $F_{\mathscr{C}}: \mathscr{R}_{\mathscr{C}} \rightarrow \mathscr{C}$ called the Reshetikhin-Turaev functor, where $\mathscr{R}_{\mathscr{C}}$ denotes the category of $\mathscr{C}$-colored ribbon graphs.

An ideal of $\mathscr{C}$ is a full subcategory of $\mathscr{C}$ whose class of objects is closed under retracts and absorbent under tensor products with arbitrary objects of $\mathscr{C}$. We denote with $\operatorname{Proj}(\mathscr{C})$ the ideal of projective objects of $\mathscr{C}$. We define the partial trace of an endomorphism $f \in \operatorname{End}_{\mathscr{C}}\left(V \otimes V^{\prime}\right)$ to be the endomorphism $\operatorname{ptr}(f) \in \operatorname{End}_{\mathscr{C}}(V)$ given by

$$
\operatorname{ptr}(f):=\left(\operatorname{id}_{V} \otimes \overrightarrow{\mathrm{ev}}_{V^{\prime}}\right) \circ\left(f \otimes \operatorname{id}_{V^{\prime *}}\right) \circ\left(\operatorname{id}_{V} \otimes \overleftarrow{\operatorname{coev}}_{V^{\prime}}\right)
$$

Then, following $[14,16]$, if $\mathscr{C}$ is a ribbon linear category over a field $\mathbb{k}$, an $m$-trace $\mathrm{t}$ on $\operatorname{Proj}(\mathscr{C})$ is a family of linear maps $\left\{\mathrm{t}_{V}: \operatorname{End}_{\mathscr{C}}(V) \rightarrow \mathbb{k} \mid V \in \operatorname{Proj}(\mathscr{C})\right\}$ satisfying:

(1) Cyclicity: $\mathrm{t}_{V}\left(f^{\prime} \circ f\right)=\mathrm{t}_{V^{\prime}}\left(f \circ f^{\prime}\right)$ for all objects $V, V^{\prime} \in \operatorname{Proj}(\mathscr{C})$ and for all morphisms $f \in \operatorname{Hom}_{\mathscr{C}}\left(V, V^{\prime}\right)$ and $f^{\prime} \in \operatorname{Hom}_{\mathscr{C}}\left(V^{\prime}, V\right)$;

(2) Partial trace: $\mathrm{t}_{V \otimes V^{\prime}}(f)=\mathrm{t}_{V}(\operatorname{ptr}(f))$ for all objects $V \in \operatorname{Proj}(\mathscr{C})$ and $V^{\prime} \in \mathscr{C}$ and for every morphism $f \in \operatorname{End}_{\mathscr{C}}\left(V \otimes V^{\prime}\right)$.

For every $V \in \operatorname{Proj}(\mathscr{C})$ we denote with $\mathrm{d}(V):=\mathrm{t}_{V}\left(\mathrm{id}_{V}\right)$ its modified dimension. We say an m-trace t on $\operatorname{Proj}(\mathscr{C})$ is non-degenerate if, for every $V \in \operatorname{Proj}(\mathscr{C})$ and $V^{\prime} \in \mathscr{C}$, the bilinear pairing $\mathrm{t}_{V}(\cdot \circ \cdot): \operatorname{Hom}_{\mathscr{C}}\left(V^{\prime}, V\right) \times \operatorname{Hom}_{\mathscr{C}}\left(V, V^{\prime}\right) \rightarrow \mathbb{k}$ is nondegenerate. 
Finally, let us fix some terminology which will be extensively used throughout the paper: every time we have a ribbon linear category $\mathscr{C}$ over a field $\mathbb{k}$, we have an associated notion of skein equivalence between formal linear combinations of $\mathscr{C}$-colored ribbon graphs. Indeed, if $(\underline{\varepsilon}, \underline{V})$ and $\left(\underline{\varepsilon}^{\prime}, \underline{V^{\prime}}\right)$ are objects of $\mathscr{R}_{\mathscr{C}}$, if $\alpha_{1}, \ldots, \alpha_{m}, \alpha_{1}^{\prime}, \ldots, \alpha_{m^{\prime}}^{\prime}$ are scalar coefficients in $\mathbb{k}$, and if $T_{1}, \ldots, T_{m}, T_{1}^{\prime}, \ldots, T_{m^{\prime}}^{\prime}$ are morphisms of $\mathscr{R}_{\mathscr{C}}$ from $(\underline{\varepsilon}, \underline{V})$ and $\left(\underline{\varepsilon^{\prime}}, \underline{V^{\prime}}\right)$, then we say two formal linear combinations $\sum_{i=1}^{m} \alpha_{i} \cdot T_{i}$ and $\sum_{i^{\prime}=1}^{m^{\prime}} \alpha_{i^{\prime}}^{\prime} \cdot T_{i^{\prime}}^{\prime}$ are skein equivalent, and we write

$$
\sum_{i=1}^{m} \alpha_{i} \cdot T_{i} \doteq \sum_{i^{\prime}=1}^{m^{\prime}} \alpha_{i^{\prime}}^{\prime} \cdot T_{i^{\prime}}^{\prime},
$$

if we have the equality $\sum_{i=1}^{m} \alpha_{i} \cdot F_{\mathscr{C}}\left(T_{i}\right)=\sum_{i^{\prime}=1}^{m^{\prime}} \alpha_{i^{\prime}}^{\prime} \cdot F_{\mathscr{C}}\left(T_{i^{\prime}}^{\prime}\right)$ under the ReshetikhinTuraev functor $F_{\mathscr{C}}: \mathscr{R}_{\mathscr{C}} \rightarrow \mathscr{C}$.

1.2. 3-Manifold invariants from non-degenerate relative pre-modular categories. Let us start by recalling the definition of relative pre-modular categories, which, as we mentioned earlier, are ribbon linear categories carrying additional structures. First of all, if $G$ is an abelian group, a compatible $G$-structure on a rigid monoidal category $\mathscr{C}$ is an equivalence of linear categories $\mathscr{C} \cong \bigoplus_{g \in G} \mathscr{C}_{g}$ for a family $\left\{\mathscr{C}_{g} \mid g \in G\right\}$ of full subcategories of $\mathscr{C}$ satisfying the following conditions: If $V \in \mathscr{C}_{g}$, then $V^{*} \in \mathscr{C}_{-g}$; If $V \in \mathscr{C}_{g}$ and $V^{\prime} \in \mathscr{C}_{g^{\prime}}$, then $V \otimes V^{\prime} \in \mathscr{C}_{g+g^{\prime}}$; If $V \in \mathscr{C}_{g}$ and $V^{\prime} \in \mathscr{C}_{g^{\prime}}$ with $g \neq g^{\prime}$, then $\operatorname{Hom}_{\mathscr{C}}\left(V, V^{\prime}\right)=0$. Remark that, if $\mathscr{C}$ is ribbon, then $\mathscr{C}_{0}$ is also ribbon. Next, if $Z$ is an abelian group, a free realization of $Z$ in a ribbon category $\mathscr{C}$ is a monoidal functor $\sigma: Z \rightarrow \mathscr{C}$, where $Z$ also denotes the discrete category over $Z$ with tensor product given by the group operation + , satisfying $\vartheta_{\sigma(k)}=\mathrm{id}_{\sigma(k)}$ for every $k \in Z$, and inducing a free action on isomorphism classes of simple objects of $\mathscr{C}$ by tensor product with $\sigma(k)$. Next, we say a subset $X$ of $G$ is symmetric if $X=-X$, and we say it is small if $G \not \subset \bigcup_{i=1}^{m}\left(g_{i}+X\right)$ for all $m \in \mathbb{N}$ and all $g_{1}, \ldots, g_{m} \in G$.

Definition 1.1 ([8]). If $G$ and $Z$ are abelian groups, and if $X \subset G$ is a small symmetric subset, then a pre-modular $G$-category relative to $(Z, X)$ is a ribbon linear category $\mathscr{C}$ over a field $\mathbb{k}$ together with a compatible $G$-structure on $\mathscr{C}$, a free realization $\sigma: Z \rightarrow \mathscr{C}_{0}$, and a non-zero m-trace t on $\operatorname{Proj}(\mathscr{C})$. These data are subject to the following conditions:

(1) Generic semisimplicity. For every $g \in G \backslash X$ the homogeneous subcategory $\mathscr{C}_{g}$ is semisimple and dominated by $\Theta\left(\mathscr{C}_{g}\right) \otimes \sigma(Z)$ for some finite set $\Theta\left(\mathscr{C}_{g}\right)=\left\{V_{i} \in \mathscr{C}_{g} \mid i \in \mathrm{I}_{g}\right\}$ of simple projective objects with epic evaluation;

(2) Compatibility. There exists a bilinear map $\psi: G \times Z \rightarrow \mathbb{k}^{*}$ such that $c_{\sigma(k), V} \circ c_{V, \sigma(k)}=\psi(g, k) \cdot \operatorname{id}_{V \otimes \sigma(k)}$ for every $g \in G$, for every $V \in \mathscr{C}_{g}$, and for every $k \in Z$.

The group $G$ is called the structure group, the group $Z$ is called the periodicity group, and the set $X$ is called the critical set of $\mathscr{C}$. Condition (1) implies that, for any $g \in G \backslash X$, every object of $\mathscr{C}_{g}$ is a direct sum of simple objects in the set $\left\{V_{i} \otimes \sigma(k) \in \mathscr{C} \mid i \in \mathrm{I}_{g}, k \in Z\right\}$. We point out that the bilinear map $\psi$ is uniquely determined by the braiding $c$ and by the free realization $\sigma$, and that, although the set of representatives $\Theta\left(\mathscr{C}_{g}\right)$ is not unique in general, its choice does not affect the following construction. In particular, both $\psi$ and $\Theta\left(\mathscr{C}_{g}\right)$ should not be considered relevant parts of the structure of $\mathscr{C}$. Relative pre-modular $G$-categories are a slight generalization of the notion of relative $G$-modular category introduced for the first time in [5], see Section 1.5 of [8] for a full discussion of the relation between the two definitions. The change in terminology is motivated by the semisimple theory, where 

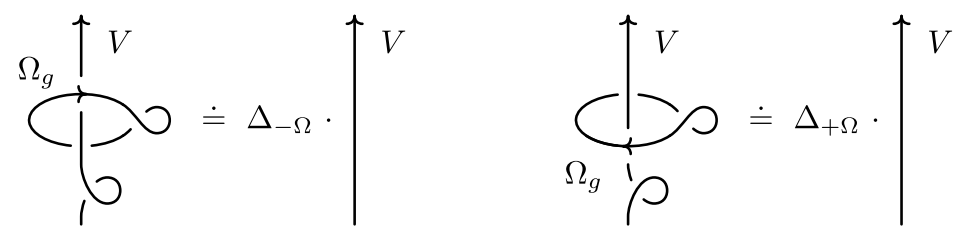

FiguRE 1. Skein equivalences defining $\Delta_{-\Omega}$ and $\Delta_{+\Omega}$.

quantum invariants are defined for any non-degenerate pre-modular category, and modularity is an additional condition ensuring the invariant extends to a TQFT. If $\mathscr{C}$ is a pre-modular $G$-category relative to $(Z, X)$ then the associated Kirby color of index $g \in G \backslash X$ is the formal linear combination of objects

$$
\Omega_{g}:=\sum_{i \in \mathrm{I}_{g}} \mathrm{~d}\left(V_{i}\right) \cdot V_{i}
$$

The name comes from Lemmas 5.9 and 5.10 of [5]. In particular, there exist constants $\Delta_{-\Omega}, \Delta_{+\Omega} \in \mathbb{k}$, called stabilization coefficients, which realize the skein equivalences of Figure 1, and which are independent of both $V \in \mathscr{C}_{g}$ and $g \in G \backslash X$. We say the relative pre-modular category $\mathscr{C}$ is non-degenerate if $\Delta_{-\Omega} \Delta_{+} \neq 0$.

In $[5,8]$ it is shown that every non-degenerate relative pre-modular category $\mathscr{C}$ gives rise to a topological invariant $\mathrm{N}_{\mathscr{C}}$ of admissible triples $(M, T, \omega)$, where $M$ is a closed 3-manifold, $T \subset M$ is a $\mathscr{C}$-colored ribbon graph, and $\omega \in H^{1}(M \backslash T ; G)$ is a compatible cohomology class, meaning that every edge $e \subset T$ is colored with an object of $\mathscr{C}_{\left\langle\omega, m_{e}\right\rangle}$ for the homology class $m_{e}$ of a positive meridian of $e$. The CGP invariant $\mathrm{N}_{\mathscr{C}}$ is defined only for admissible triples $(M, T, \omega)$, which are triples such that every component of $M$ contains either a projective edge of $T$, that is an edge of $T$ whose color is a projective object of $\mathscr{C}$, or a generic curve for $\omega$, that is an embedded closed oriented curve whose homology class is sent to $G \backslash X$ by $\omega$. Its definition uses computable surgery presentations in $S^{3}$, which are surgery presentations $L=L_{1} \cup \ldots \cup L_{\ell}$ of $M$ satisfying $\left\langle\omega, m_{j}\right\rangle \in G \backslash X$ for all integers $1 \leqslant j \leqslant \ell$, where $m_{j}$ denotes the homology class of a meridian of the component $L_{j}$. We interpret a surgery presentation of $M$ which is computable with respect to some decoration $(T, \omega)$ as a $\mathscr{C}$-colored ribbon graph by arbitrarily choosing orientations, and by labeling every component with the corresponding Kirby color, with index prescribed by the evaluation of $\omega$ against the homology class of a positive meridian. This is a technical complication, because arbitrary surgery presentations are not computable in general. Computable surgery presentations do exist for admissible decorated closed 3-manifolds, but only up to replacing admissible decorations via certain operations called projective and generic stabilizations, see Section 3.1 of [8]. The idea is to build $\mathrm{N}_{\mathscr{C}}$ out of a renormalized invariant $F_{\mathscr{C}}^{\prime}$ of admissible closed $\mathscr{C}$-colored ribbon graphs which combines the Reshetikhin-Turaev functor $F_{\mathscr{C}}$ on $\mathscr{R}_{\mathscr{C}}$ with the m-trace t on $\operatorname{Proj}(\mathscr{C})$. We say a closed $\mathscr{C}$-colored ribbon graph is admissible if one of his edges is projective. Example 3 of Section 1.5 in [18] (see also $[19,14,20])$ implies the formula

$$
F_{\mathscr{C}}^{\prime}(T):=\mathrm{t}_{V}\left(F_{\mathscr{C}}\left(T_{V}\right)\right)
$$

defines a topological invariant of the admissible closed $\mathscr{C}$-colored ribbon graph $T$, where $V \in \mathscr{C}$ is projective, and where $T_{V}$ is a cutting presentation of $T$, i.e. an endomorphism of $(+, V)$ in $\mathscr{R}_{\mathscr{C}}$ whose trace is $T$. Then, for a fixed choice of a square root $\mathscr{D}_{\Omega} \in \mathbb{k}$ of $\Delta_{-\Omega} \Delta_{+\Omega}$, the formula

$$
\mathrm{N}_{\mathscr{C}}(M, T, \omega):=\mathscr{D}_{\Omega}^{-1-\ell} \delta_{\Omega}^{-\sigma(L)} F_{\mathscr{C}}^{\prime}(L \cup \tilde{T})
$$


defines a topological invariant of the admissible triple $(M, T, \omega)$ thanks to Proposition 3.1 of [8], where $L \subset S^{3}$ is an $\ell$-component surgery presentation of $M$ of signature $\sigma(L)$ which is computable with respect to an admissible decoration $(\tilde{T}, \tilde{\omega})$ obtained from $(T, \omega)$ by performing projective or generic stabilization, and where $\delta_{\Omega}=\mathscr{D}_{\Omega} / \Delta_{-\Omega}$.

1.3. $2+1-T Q F T s$ from relative modular categories. A stronger non-degeneracy condition is required in order to extend CGP invariants to graded TQFTs.

Definition $1.2([8])$. A pre-modular $G$-category $\mathscr{C}$ relative to $(Z, X)$ is relative modular if there exists a relative modularity parameter $\zeta_{\Omega} \in \mathbb{k}^{*}$ realizing the skein equivalence of Figure 2 for all $g, h \in G \backslash X$ and for all $i, j \in \mathrm{I}_{g}$.

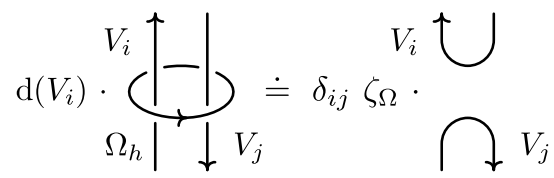

FigURE 2. Relative modularity condition.

This condition automatically implies non-degeneracy, because the relative modularity parameter satisfies $\zeta_{\Omega}=\Delta_{-\Omega} \Delta_{+}$, see [8]. When $\mathscr{C}$ is relative modular then, as explained in Section 6.2 of [8], $\mathrm{N}_{\mathscr{C}}$ extends to a $Z$-graded $2+1$-TQFT $\mathbb{V}_{\mathscr{C}}^{Z}: \check{C}^{\prime} b_{\mathscr{C}}^{G} \rightarrow \operatorname{Vect}_{\mathbb{k}}^{Z}$ via a $Z$-graded refinement of the universal construction of [3], where $\operatorname{Cob}_{\mathscr{C}}^{G}$ is the category of admissible cobordisms of dimension $2+1$, and where $\operatorname{Vect}_{\mathbb{k}}^{Z}$ is the category of $Z$-graded vector spaces. More precisely, an object of $\mathrm{Cob}_{\mathscr{C}}^{G}$ is a 5 -tuple $\mathbb{\Sigma}=(\Sigma, P, \vartheta, B, \mathscr{L})$, where $\Sigma$ is a closed surface, where $P \subset \Sigma$ is a $\mathscr{C}$-colored ribbon set, where $\vartheta \in H^{1}(\Sigma \backslash P ; G)$ is a compatible cohomology class, where $B \subset \Sigma \backslash P$ is a finite set composed of exactly one base point in every connected component of $\Sigma$, and where $\mathscr{L} \subset H_{1}(\Sigma ; \mathbb{R})$ is a Lagrangian subspace. A morphism of $\operatorname{Cob}_{\mathscr{C}}^{G}$ from $(\Sigma, P, \vartheta, B, \mathscr{L})$ to $\left(\Sigma^{\prime}, P^{\prime}, \vartheta^{\prime}, B^{\prime}, \mathscr{L}^{\prime}\right)$ is an equivalence class of admissible 4-tuples $\mathbb{M}=(M, T, \omega, n)$, where $M$ is a 3 -dimensional cobordism from $\Sigma$ to $\Sigma^{\prime}$, where $T \subset M$ is a $\mathscr{C}$-colored ribbon graph from $P$ to $P^{\prime}$, where $\omega \in H^{1}\left(M \backslash T, B \cup B^{\prime} ; G\right)$ is a compatible relative cohomology class restricting to $\vartheta$ and $\vartheta^{\prime}$ on the incoming and outgoing boundary of $M$ respectively, and where $n \in \mathbb{Z}$ is a signature defect. A 4-tuple $(M, T, \omega, n)$ is admissible if every component of $M$ which is disjoint from the incoming boundary $\partial_{-} M$ contains either a projective edge of $T$, or a generic curve for $\omega$, and two 4-tuples $(M, T, \omega, n)$ and $\left(M^{\prime}, T^{\prime}, \omega^{\prime}, n^{\prime}\right)$ are equivalent if $n=n^{\prime}$, and if there exists a positive diffeomorphism $f: M \rightarrow M^{\prime}$ which preserves boundary identifications and satisfies $f(T)=T^{\prime}$ and $f^{*}\left(\omega^{\prime}\right)=\omega$. Then, $\mathrm{N}_{\mathscr{C}}$ can be extended to an invariant of closed morphisms of $\mathrm{Cob}_{\mathscr{C}}^{G}$ by setting

$$
\mathrm{N}_{\mathscr{C}}(M, T, \omega, n):=\delta_{\Omega}^{n} \mathrm{~N}_{\mathscr{C}}(M, T, \omega) .
$$

Remark that the category $\check{C o b}_{\mathscr{C}}^{G}$ thus obtained is not rigid, as objects $(\Sigma, P, \vartheta, \mathscr{L})$ such that $P$ does not contain any projective point and such that $\vartheta$ does not admit any generic curve are not dualizable.

State spaces associated with objects of $\check{C o b}_{\mathscr{C}}^{G}$ by the $Z$-graded TQFT $\mathbb{V}_{\mathscr{C}}^{Z}$ can be described in skein theoretical terms in all degrees. We have two relevant notions of skein equivalence between morphisms of $\check{C} b_{\mathscr{C}}^{G}$ from $\mathbb{\Sigma}$ to $\mathbb{\Sigma}^{\prime}$, one which is local, the other which is not. Indeed, we say a formal linear combination of morphisms of $\mathrm{Cob}_{\mathscr{C}}^{G}$ from $\mathbb{Z}$ to $\mathbb{Z}^{\prime}$ is a local skein relation if it can be written in the form

$$
\sum_{i=1}^{m} \alpha_{i} \cdot \mathbb{M}_{P} \circ\left(\left(D^{3}, T_{i}, \omega_{i}, 0\right) \sqcup \mathrm{id}_{\mathbb{\Sigma}}\right)
$$


for some coefficients $\alpha_{1}, \ldots, \alpha_{m} \in \mathbb{k}$, for some $\mathscr{C}$-colored ribbon set $P \subset S^{2}$ with at least one point labeled by a projective object, for some morphism $\mathbb{M}_{P}$ of Cob $_{\mathscr{C}}^{G}$ from $\left(S^{2}, P, \vartheta,\{0\}\right) \sqcup \mathbb{Z}$ to $\mathbb{Z}^{\prime}$, and for some $\mathscr{C}$-colored ribbon graphs $T_{1}, \ldots, T_{m} \subset D^{3}$ from $\varnothing$ to $P$ satisfying

$$
\sum_{i=1}^{m} \alpha_{i} \cdot f_{D^{3}}\left(T_{i}\right) \doteq 0
$$

with respect to some embedding $f_{D^{3}}: D^{3} \hookrightarrow \mathbb{R}^{2} \times I$ mapping $P$ into $\mathbb{R}^{2} \times\{1\}$. Here the cohomology classes $\vartheta$ and $\omega_{1}, \ldots, \omega_{m}$ are uniquely determined by $P$ and by $T_{1}, \ldots, T_{m}$ respectively. On the other hand, we say a formal linear combination of morphisms of $\mathrm{Cob}_{\mathscr{C}}^{G}$ from $\mathbb{\Sigma}$ to $\mathbb{\Sigma}^{\prime}$ is a non-local skein relation if it can be written in the form

$$
\left(M, T \cup K, j^{*}(\omega), n\right)-\psi\left(\left\langle\omega, \ell_{K}\right\rangle, k\right) \cdot(M, T, \omega, n)
$$

for some $k \in Z$ and for some framed $\operatorname{knot} K \subset M \backslash T$ of color $\sigma(k)$, where $j^{*}$ is induced by the inclusion of $M \backslash(T \cup K)$ into $M \backslash T$, and where $\ell_{K}$ denotes the homology class of $K$ in $H_{1}(M \backslash T ; \mathbb{Z})$. Then, if $\mathbb{Z}=(\Sigma, P, \vartheta, B, \mathscr{L})$ is an object of $\mathrm{Cob}_{\mathscr{C}}^{G}$, and if $M$ is a 3-dimensional cobordism from $\varnothing$ to $\Sigma$, the admissible skein module $\breve{\mathrm{S}}(M ; \mathbb{\Sigma})$ is the quotient, induced by both local and non-local skein relations, of the free vector space $\mathscr{V}(M ; \mathbb{Z})$ generated by all pairs $(T, \omega)$ such that $(M, T, \omega, 0)$ is a morphism of $\operatorname{Cob}_{\mathscr{C}}^{G}$ from $\varnothing$ to $\mathbb{Z}$. The class of a generator $(T, \omega)$ of $\mathscr{V}(M ; \mathbb{Z})$ in $\check{\mathrm{S}}(M ; \mathbb{Z})$ is denoted $[T, \omega]$. The proof of Proposition 4.5 in [2] can be adapted to show that if $\Sigma$ and $M$ are both connected, then $\check{\mathrm{S}}(M ; \mathbb{Z})$ is a finite-dimensional vector space. On the other hand, if $M^{\prime}$ is a 3-dimensional cobordism from $\Sigma$ to $\varnothing$, we denote with $\mathscr{V}^{\prime}\left(M^{\prime} ; \mathbb{\Sigma}\right)$ the free vector space generated by all pairs $\left(T^{\prime}, \omega^{\prime}\right)$ such that $\left(M^{\prime}, T^{\prime}, \omega^{\prime}, 0\right)$ is a morphism of $\check{C}_{\mathscr{C}}^{G}$ from $\mathbb{\Sigma}$ to $\varnothing$. Then, for every $k \in Z$, the degree $k$ state space $\mathbb{V}_{\mathscr{C}}^{k}(\mathbb{Z})$ of a connected object $\mathbb{Z}$ of $\check{C}_{\mathscr{C}}^{G}$ satisfies

$$
\mathbb{V}_{\mathscr{C}}^{k}(\mathbb{\Sigma}) \cong \check{\mathrm{S}}\left(M ; \mathbb{\Sigma} \sqcup \mathbb{S}_{-k}^{2}\right) / \mathscr{V}^{\prime}\left(M^{\prime} ; \mathbb{\Sigma} \sqcup \mathbb{S}_{-k}^{2}\right)^{\perp}
$$

with respect to the pairing $\langle\cdot, \cdot\rangle_{\mathbb{\mathbb { }} \sqcup \mathbb{S}_{-k}^{2}}: \mathscr{V}^{\prime}\left(M^{\prime} ; \mathbb{Z} \sqcup \mathbb{S}_{-k}^{2}\right) \otimes \check{\mathrm{S}}\left(M ; \mathbb{\mathbb { }} \sqcup \mathbb{S}_{-k}^{2}\right) \rightarrow \mathbb{k}$ defined by

$$
\left\langle\left(T^{\prime}, \omega^{\prime}\right),[T, \omega]\right\rangle_{\mathbb{\Sigma} \sqcup \mathbb{S}_{-k}^{2}}:=\mathrm{N}_{\mathscr{C}}\left(\left(M^{\prime}, T^{\prime}, \omega^{\prime}, 0\right) \circ(M, T, \omega, 0)\right),
$$

where $M$ is a connected 3-dimensional cobordism from $\varnothing$ to $\Sigma \sqcup S^{2}$, where $M^{\prime}$ is a connected 3-dimensional cobordism from $\Sigma \sqcup S^{2}$ to $\varnothing$, and where the object

$$
\mathbb{S}_{-k}^{2}=\left(S^{2}, P_{\left(\left(+, V_{0}\right),(+, \sigma(-k)),\left(-, V_{0}\right)\right)}, \vartheta_{\left(\left(+, V_{0}\right),(+, \sigma(-k)),\left(-, V_{0}\right)\right)}, B,\{0\}\right)
$$

of $\check{\mathrm{Cob}}_{\mathscr{C}}^{G}$ is determined by the $\mathscr{C}$-colored ribbon set $P_{\left(\left(+, V_{0}\right),(+, \sigma(-k)),\left(-, V_{0}\right)\right)} \subset S^{2}$ composed of three points in standard positions with orientations and colors specified by their subscript for some $g_{0} \in G \backslash X$ and for some $V_{0} \in \Theta\left(\mathscr{C}_{g_{0}}\right)$. Remark that an explicit characterization of these quotients can sometimes be achieved. For instance, if $P \subset S^{2}$ is a $\mathscr{C}$-colored ribbon set composed of a single positive point of color $F_{\mathscr{C}}(P) \in \operatorname{Proj}\left(\mathscr{C}_{0}\right)$, then, thanks to Remark 7.2 of [8], the $Z$-graded state space $\mathbb{V}_{\mathscr{C}}^{Z}\left(\mathbb{S}_{P}^{2}\right)$ of the object $\mathbb{S}_{P}^{2}=\left(S^{2}, P, 0,\{0\}\right)$ of $\check{C o b}_{\mathscr{C}}^{G}$ satisfies

$$
\mathbb{V}_{\mathscr{C}}^{Z}\left(\mathbb{S}_{P}^{2}\right) \cong \mathbb{H} \operatorname{lom}_{\mathscr{C}}\left(\mathbb{1}, F_{\mathscr{C}}(P)\right)
$$

where for all $V, V^{\prime} \in \mathscr{C}$ we denote with $\mathbb{H}_{\mathscr{C}}\left(V, V^{\prime}\right)$ the $Z$-graded vector space whose space of degree $k$ vectors is given by $\operatorname{Hom}_{\mathscr{C}}\left(V, V^{\prime} \otimes \sigma(-k)\right)$ for every $k \in Z$. See also Proposition 7.16 of [8] for a description, in terms of homogeneous colorings of trivalent graphs, of the $Z$-graded state space of generic surfaces in $\mathrm{Cob}_{\mathscr{C}}^{G}$. 
1.4. 3-Manifold invariants from finite-dimensional non-degenerate unimodular ribbon Hopf algebras. Next, let us move on to the renormalized Hennings theory. We start by fixing our notation for Hopf algebras, and by recalling some crucial definitions and results. If $\mathbb{k}$ is a field, a finite-dimensional ribbon Hopf algebra $H$ is a finite-dimensional vector space over $\mathbb{k}$ endowed with a multiplication $m: H \otimes H \rightarrow H$, a unit $\eta: \mathbb{k} \rightarrow H$, a coproduct $\Delta: H \rightarrow H \otimes H$, a counit $\varepsilon: H \rightarrow \mathbb{k}$, an antipode $S: H \rightarrow H$, an R-matrix $R=\sum_{i=1}^{r} a_{i} \otimes b_{i} \in H \otimes H$, and a ribbon element $v$ in the center of $H$, see [31] for a list of the axioms these structure maps and elements are subject to. We use the notation $m(x \otimes y)=x y$ for every $x \otimes y \in H \otimes H$ and $\eta(1)=1$, and we denote with $u=\sum_{i=1}^{r} S\left(b_{i}\right) a_{i} \in H$ the Drinfeld element, and with $g=u v^{-1} \in H$ the pivotal element associated with the ribbon structure of $H$. As a consequence of finite-dimensionality, $H$ admits a right integral $\lambda \in H^{*}$ and a left cointegral $\Lambda \in H$ which are unique up to scalar, and we can fix a pair satisfying $\lambda(\Lambda)=1$. The Hopf algebra $H$ is non-degenerate if the stabilization coefficients $\Delta_{-\lambda}:=\lambda(v)$ and $\Delta_{+\lambda}:=\lambda\left(v^{-1}\right)$ satisfy $\Delta_{-\lambda} \Delta_{+\lambda} \neq 0$, and it is unimodular if the left cointegral $\Lambda$ is two-sided, meaning that it is also a right cointegral. The category $\mathscr{C}=H$-mod of finite-dimensional left $H$-modules is a ribbon linear category, with evaluation and coevaluation morphisms given by

$$
\begin{array}{rllllll}
\overleftarrow{\mathrm{ev}}_{V}: V^{*} \otimes V & \rightarrow \mathbb{k} & \overleftarrow{\operatorname{coev}}_{V}: & \mathbb{k} & \rightarrow & V \otimes V^{*} \\
f \otimes v & \mapsto f(v) & & & \mapsto \sum_{i=1}^{n} v_{i} \otimes f_{i} \\
\overrightarrow{\mathrm{ev}}_{V}: V \otimes V^{*} & \rightarrow & \mathbb{k} & \overrightarrow{\operatorname{coev}}_{V}: & \mathbb{k} & \rightarrow & V^{*} \otimes V \\
v \otimes f & \mapsto f\left(\rho_{V}(g)(v)\right) & & 1 & \mapsto \sum_{i=1}^{n} f_{i} \otimes \rho_{V}\left(g^{-1}\right)\left(v_{i}\right)
\end{array}
$$

for every left $H$-module $V$ with basis $\left\{v_{1}, \ldots, v_{n}\right\}$ and dual basis $\left\{f_{1}, \ldots, f_{n}\right\}$, and with braiding morphisms given by

$$
\begin{array}{rlc}
c_{V, V^{\prime}}: V \otimes V^{\prime} & \rightarrow \quad V^{\prime} \otimes V \\
v \otimes v^{\prime} & \mapsto \sum_{i=1}^{r} \rho_{V^{\prime}}\left(b_{i}\right)\left(v^{\prime}\right) \otimes \rho_{V}\left(a_{i}\right)
\end{array}
$$

for all left $H$-modules $V$ and $V^{\prime}$. Thanks to Theorem 1 of [1], $\mathscr{C}$ admits an mtrace t on the ideal of projective $H$-modules $\operatorname{Proj}(\mathscr{C})$, which is unique up to scalar and uniquely determined by the condition $\mathrm{t}_{H}(f)=\lambda(g f(1))$ for all $f \in \operatorname{End}_{\mathscr{C}}(H)$, where $H \in \operatorname{Proj}(\mathscr{C})$ denotes the regular representation of $H$. Furthermore, $\mathrm{t}$ is non-degenerate.

In [9] it is shown that every finite-dimensional non-degenerate ribbon Hopf algebra $H$ gives rise to a topological invariant $\mathrm{H}_{\mathscr{C}}^{\prime}$ of admissible pairs $(M, T)$, where $M$ is a closed 3-manifold, and $T \subset M$ is a $\mathscr{C}$-colored bichrome graph. The latter are $\mathscr{C}$-colored ribbon graphs carrying a set of specified edges, and their name comes from the fact that we think about special edges as being red, while the rest of the graph is blue. Red edges can only be colored with the regular representation $H$, and they can only intersect coupons in a prescribed way: for every coupon of a bichrome graph there exists an integer $k \geqslant 0$ such that the first $k$ input edges are incoming and red, the first $k$ output edges are outgoing and red, while all the other ones are blue. Such a coupon is colored with a morphism in the $k$-th stabilized subcategory $[k] \mathscr{C}$ of $\mathscr{C}$, which is the category whose objects have the form $[k] V:=H^{\otimes k} \otimes V \in \mathscr{C}$ for some $V \in \mathscr{C}$, and whose morphisms have the form $\sum_{i=1}^{m} L_{x_{i}} \otimes f_{i} \in \operatorname{Hom}_{\mathscr{C}}\left([k] V,[k] V^{\prime}\right)$ for some left translation $L_{x_{i}} \in \operatorname{End}_{\mathbb{k}}\left(H^{\otimes k}\right)$ by $x_{i} \in H^{\overline{\otimes k}}$ and for some linear map $f_{i} \in \operatorname{Hom}_{\mathbb{k}}\left(V, V^{\prime}\right)$. The ribbon category $\mathscr{R}_{\lambda}$ $\overline{\text { of }} \mathscr{C}$-colored bichrome graphs provides a graphical calculus which is formalized by 
the Hennings-Reshetikhin-Turaev functor $F_{\lambda}: \mathscr{R}_{\lambda} \rightarrow \mathscr{C}$ introduced in Proposition 2.5 of [9]. By definition, $F_{\lambda}$ coincides with the Reshetikhin-Turaev functor in the absence of red edges, it coincides with the Hennings invariant in the absence of blue edges, and it coherently combines the two behaviors for general $\mathscr{C}$-colored bichrome graphs. Remark that $F_{\lambda}$ yields a notion of skein equivalence between formal linear combinations of $\mathscr{C}$-colored bichrome graphs in the same way $F_{\mathscr{C}}$ does for $\mathscr{C}$-colored ribbon graphs. This way, $\mathscr{R}_{\mathscr{C}}$ is naturally identified with the subcategory of $\mathscr{R}_{\lambda}$ whose morphisms are entirely blue. The renormalized Hennings invariant $\mathrm{H}_{\mathscr{C}}^{\prime}$ is then defined only for admissible pairs $(M, T)$, which are pairs such that every component of $M$ contains a projective blue edge of $T$. Its definition uses surgery presentations in $S^{3}$, which we interpret as $\mathscr{C}$-colored bichrome graphs by arbitrarily choosing orientations, by labeling every component with the regular representation $H$, and by taking them to be red. The idea is to build $\mathrm{H}_{\mathscr{C}}^{\prime}$ out of a renormalized invariant $F_{\lambda}^{\prime}$ of admissible closed $\mathscr{C}$-colored bichrome graphs which combines the Hennings-Reshetikhin-Turaev functor $F_{\lambda}$ on $\mathscr{R}_{\lambda}$ with the m-trace t on $\operatorname{Proj}(\mathscr{C})$. We say a closed $\mathscr{C}$-colored bichrome graph is admissible if one of his blue edges is projective. The formula

$$
F_{\lambda}^{\prime}(T):=\mathrm{t}_{V}\left(F_{\lambda}\left(T_{V}\right)\right)
$$

defines a topological invariant of the admissible closed $\mathscr{C}$-colored bichrome graph $T$ thanks to Theorem 2.7 of [9], where $V$ is a projective object of $\mathscr{C}$, and where $T_{V}$ is a cutting presentation of $T$, meaning an endomorphism of $(+, V)$ in $\mathscr{R}_{\lambda}$ whose trace is $T$. Then, for a fixed choice of a square root $\mathscr{D}_{\lambda} \in \mathbb{k}$ of $\Delta_{-\lambda} \Delta_{+\lambda}$, the formula

$$
\mathrm{H}_{\mathscr{C}}^{\prime}(M, T):=\mathscr{D}_{\lambda}^{-1-\ell} \delta_{\lambda}^{-\sigma(L)} F_{\lambda}^{\prime}(L \cup T)
$$

defines a topological invariant of the admissible pair $(M, T)$ thanks to Theorem 2.9 of [9], where $L \subset S^{3}$ is an $\ell$-component surgery presentation of $M$ of signature $\sigma(L)$, and where $\delta_{\lambda}=\mathscr{D}_{\lambda} / \Delta_{-\lambda}$.

1.5. 2+1-TQFTs from finite-dimensional factorizable ribbon Hopf algebras. A finite-dimensional ribbon Hopf algebra $H$ with R-matrix $R=\sum_{i=1}^{r} a_{i} \otimes b_{i}$ is factorizable if the Drinfeld map $\psi_{H}: H^{*} \rightarrow H$, which is defined by

$$
\psi_{H}(f):=\sum_{i, j=1}^{r} f\left(b_{j} a_{i}\right) \cdot a_{j} b_{i}
$$

for every $f \in H^{*}$, is an isomorphism. This condition implies both non-degeneracy and unimodularity, see [21] and [31]. When $H$ is factorizable then, as explained in Section 3 of [9], $\mathrm{H}_{\mathscr{C}}^{\prime}$ extends to a $2+1$-TQFT $\mathrm{V}_{\mathscr{C}}:$ Cob $_{\mathscr{C}} \rightarrow$ Vect $_{\mathbb{k}}$ via the universal construction of [3], where $\mathrm{Cob}_{\mathscr{C}}$ is the category of admissible cobordisms of dimension $2+1$. More precisely, an object of $\check{C}_{\mathscr{C}}$ is a triple $\mathbb{\Sigma}=(\Sigma, P, \mathscr{L})$, where $\Sigma$ is a closed surface, where $P \subset \Sigma$ is a blue $\mathscr{C}$-colored ribbon set, and where $\mathscr{L} \subset H_{1}(\Sigma ; \mathbb{R})$ is a Lagrangian subspace. A morphism of $\mathrm{Cob}_{\mathscr{C}}$ from $(\Sigma, P, \mathscr{L})$ to $\left(\Sigma^{\prime}, P^{\prime}, \mathscr{L}^{\prime}\right)$ is an equivalence class of admissible triples $\mathbb{M}=(M, T, n)$, where $M$ is a 3-dimensional cobordism from $\Sigma$ to $\Sigma^{\prime}$, where $T \subset M$ is a $\mathscr{C}$-colored bichrome graph from $P$ to $P^{\prime}$, and where $n \in \mathbb{Z}$ is a signature defect. A triple $(M, T, n)$ is admissible if every component of $M$ which is disjoint from the incoming boundary $\partial_{-} M$ contains a projective blue edge of $T$, and two triples $(M, T, n)$ and $\left(M^{\prime}, T^{\prime}, n^{\prime}\right)$ are equivalent if $n=n^{\prime}$, and if there exists a positive diffeomorphism $f: M \rightarrow M^{\prime}$ which preserves boundary identifications and satisfies $f(T)=T^{\prime}$. Then, $\mathrm{H}_{\mathscr{C}}^{\prime}$ can be extended to an invariant of closed morphisms of Cob $_{\mathscr{C}}$ by setting

$$
\mathrm{H}_{\mathscr{C}}^{\prime}(M, T, n):=\delta_{\lambda}^{n} \mathrm{H}_{\mathscr{C}}^{\prime}(M, T) .
$$


Remark that the category $\mathrm{Cob}_{\mathscr{C}}$ thus obtained is not rigid, as objects $(\Sigma, P, \mathscr{L})$ such that $P$ does not contain any projective blue point are not dualizable.

State spaces associated with objects of $\check{C o b}_{\mathscr{C}}$ by the TQFT $\mathrm{V}_{\mathscr{C}}$ can be presented as quotients of admissible skein modules, just like we did in the CGP case, and of course this time only local skein relations are needed. However, they can also be efficiently described in terms of the dual coadjoint $H$-module $X$, which is the vector space $H$ equipped with the action $\rho_{X}(h)(x):=h_{(2)} x S^{-1}\left(h_{(1)}\right)$ for all $h \in H$ and $x \in X$. Indeed, if $V$ is a left $H$-module with action $\rho_{V}: H \rightarrow \operatorname{End}_{\mathbb{k}}(V)$, we can consider its subspace of $H$-invariant vectors, which is defined as

$$
V^{H}:=\left\{v \in V \mid \rho_{V}(h)(v)=\varepsilon(h) \cdot v \forall h \in H\right\} .
$$

Remark that we have an obvious isomorphism between $\operatorname{Hom}_{\mathscr{C}}(\mathbb{1}, V)$ and $V^{H}$ sending $f$ to $f(1)$. Then, if $\Sigma_{g}$ is a closed surface of genus $g \in \mathbb{N}$, and if $P_{V} \subset \Sigma_{g}$ is a single positive framed blue point of color $V \in \mathscr{C}$, it follows directly from Corollary 3.21

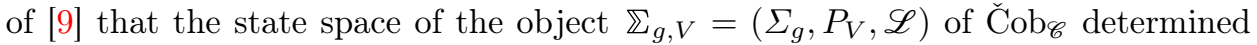
by any arbitrary Lagrangian $\mathscr{L} \subset H_{1}\left(\Sigma_{g} ; \mathbb{R}\right)$ satisfies

$$
\mathrm{V}_{\mathscr{C}}\left(\mathbb{\Sigma}_{g, V}\right) \cong\left(\left(V^{*} \otimes X^{\otimes g}\right)^{H}\right)^{*} .
$$

1.6. Main results. As we mentioned earlier, this paper contains two main results related to the non-semisimple constructions we just recalled. The first one concerns the existence of a family of graded TQFTs, as well as graded ETQFTs, for the CGP theory. The setting is provided by unrolled quantum groups at odd roots of unity. More precisely, in Subsection 2.2 we recall the definition, for every simple complex Lie algebra $\mathfrak{g}$ of rank $n$ and dimension $2 N+n$, of a particular quantum deformation, denoted $U_{q}^{H}(\mathfrak{g})$, of the enveloping algebra $U(\mathfrak{g})$ for $q=e^{\frac{2 \pi i}{r}}$, where $r \geqslant 3$ is an odd integer which is required not to be a multiple of 3 when $\mathfrak{g}=\mathfrak{g}_{2}$. These unrolled quantum groups are quite different from the ones which usually underlie quantum constructions in low dimensional topology. For instance, they are infinite dimensional: indeed, they are generated by $\left\{E_{i}, F_{i}, H_{i}, K_{i}, K_{i}^{-1} \mid 1 \leqslant i \leqslant n\right\}$, but while generators $E_{i}$ and $F_{i}$, as well as their induced root vectors, are set to be nilpotent, generators $K_{i}$ and $K_{i}^{-1}$ are not required to be quasi-unipotent. This produces a representation theory in which weights are allowed to take arbitrary complex values, instead of integral ones. As a consequence, we need to be careful when it comes to defining braidings of representations. Indeed, we need the presence of generators $H_{i}$, which should be thought of a logarithms of generators $K_{i}$. This exponential relation is not set at the level of the quantum group, but we restrict to representations where it is satisfied. More precisely, we focus on the full subcategory $\mathscr{C}^{H}$ of finite-dimensional representations of $U_{q}^{H} \mathfrak{g}$ where the action of generators $H_{i}$ is diagonalizable, and where the action of generators $K_{i}$ is obtained by exponentiating. This category is non-semisimple, and it was studied in detail in [17]: a full subcategory $\mathscr{D}^{\vartheta}$ of $\mathscr{C}^{H}$ was proven to be ribbon, and the equality $\mathscr{D}^{\vartheta}=\mathscr{C}^{H}$ was conjectured. In [5], it was shown that $\mathscr{D}^{\vartheta}$ is non-degenerate relative pre-modular, and thus yields a quantum invariant $\mathrm{N}_{\mathscr{C}}$ of admissible decorated 3-manifolds. In [18], the conjecture was proven: $\mathscr{C}^{H}$ is a relative pre-modular category. Its structure group $G$ is given by $\mathfrak{h}^{*} / \Lambda_{R}$, where $\mathfrak{h}$ is a Cartan subalgebra of $\mathfrak{g}$ with root lattice $\Lambda_{R}$. Its periodicity group $Z$ is given by $\Lambda_{R} \cap\left(r \cdot \Lambda_{W}\right)$, where $\Lambda_{W}$ denotes the weight lattice. Its critical set $X$ is given by $\left\{[\xi] \in \mathfrak{h}^{*} / \Lambda_{R} \mid \exists \alpha \in \Phi_{+}: 2\langle\alpha, \xi\rangle \in \mathbb{Z}\right\}$, where $\Phi_{+}$is a set of positive roots of $\mathfrak{g}$. The following is our first main result, which implies, as an immediate consequence, the existence of a $Z$-graded ETQFT in dimension $1+1+1$ extending the quantum invariant $\mathrm{N}_{\mathscr{C}^{H}}$.

Theorem 1.3. The category $\mathscr{C}^{H}$ is relative modular. 
The second main result of this paper builds a bridge between this family of quantum invariants and the renormalized Hennings ones coming from the corresponding small quantum groups, under the additional assumption that $\operatorname{gcd}(r, \operatorname{det}(A))=1$, where $A$ denotes the Cartan matrix of $\mathfrak{g}$. Indeed, in Subsection 2.1 we recall the definition of a more classical quantum version of $U(\mathfrak{g})$, denoted $\bar{U}_{q}(\mathfrak{g})$, again for $q=e^{\frac{2 \pi i}{r}}$. These small quantum groups are far better known: they are finitedimensional, as generators $K_{i}$ and $K_{i}^{-1}$ are set to be quasi-unipotent, they are ribbon and factorizable, and thus they yield TQFTs in dimension $2+1$. The category $\overline{\mathscr{C}}$ of finite-dimensional representations of $\bar{U}_{q} \mathfrak{g}$ is still non-semisimple, but all weights take integral values. Indeed, we have a very natural forgetful functor $\Phi_{\mathscr{C}}$ from the full subcategory $\mathscr{C}_{[0]}^{H}$ of $\mathscr{C}^{H}$ whose objects have all weights in $\Lambda_{R}$ to $\overline{\mathscr{C}}$ : the image $\bar{V}$ of an object $V$ of $\mathscr{C}_{[0]}^{H}$ is simply defined by forgetting the action of generators $H_{i}$. The technical condition $\operatorname{gcd}(r, \operatorname{det}(A))=1$ ensures $\Phi_{\mathscr{C}}$ is essentially surjective. Furthermore, $\Phi_{\mathscr{C}}$ immediately induces a functor $\Phi_{\mathscr{R}}$ from $\mathscr{R}_{\mathscr{C}_{[0]}^{H}}$ to $\mathscr{R}_{\overline{\mathscr{C}}}$ : the image $\bar{T}$ of a morphism $T$ of $\mathscr{R}_{\mathscr{C}_{[0]}^{H}}$ is simply defined by applying the forgetful functor $\Phi_{\mathscr{C}}$ to all its colors.

Theorem 1.4. If $M$ is a closed 3-manifold and $T \subset M$ is an admissible $\mathscr{C}_{[0]}^{H}$-colored ribbon graph, then

$$
\mathrm{N}_{\mathscr{C} H}(M, T, 0)=\mathrm{H}_{\overline{\mathscr{C}}}^{\prime}(M, \bar{T}) .
$$

We point out that each invariant depends on the choice of a square root of the product of the stabilization coefficients for the corresponding version of the quantum group. Theorem 1.4 requires a coherent choice of these square roots in the two theories, otherwise a sign will appear in the relation. Remark also that a great advantage of the renormalized Hennings theory is the absence of many technical complications which characterize the CGP one. For instance, arbitrary surgery presentations can be used to define and compute quantum invariants associated with small quantum groups, as we have no computability condition. Therefore, Theorem 1.4 gives a convenient alternative formulation for the CGP invariants associated with unrolled quantum groups, at least in the case of 3-manifolds decorated with the trivial cohomology class.

Let us end this introduction with a final comment about our choice for the setting of Theorem 1.4. When we started writing these results, the renormalized Hennings construction had only been developed in the case of finite-dimensional factorizable ribbon Hopf algebras. As a consequence, a comparison with the CGP construction had to take place in this context. The classification of finite-dimensional factorizable quantum groups is not so easy, see [25], and we choose for simplicity to limit ourselves to the smallest set of Cartan generators $\left\{K_{i} \mid 1 \leqslant i \leqslant n\right\}$ at odd roots of unity. However, a recent generalization of the renormalized Hennings construction allows us to consider arbitrary modular categories, in the non-semisimple sense, as building blocks for non-semisimple 2+1-TQFTs [10]. This larger setting encompasses examples of ribbon categories coming from the representation theory of restricted quantum groups at even roots of unity. Indeed, while some of these Hopf algebras might not be braided [24, 25], a suitable modification of their coalgebra structure produces quasi-Hopf deformations which admit a ribbon structure $[6,13,30]$. For what concerns unrolled quantum groups, even roots of unity have been less studied due to several technical difficulties which produce fascinating but complicated phenomena in the theory. It would be very interesting to generalize Theorem 1.4 to this setting, and possibly to even larger ones. 


\section{QuANTUM GROUPS AT ODD ROOTS OF UNITY}

In this section we recall definitions of small and unrolled quantum groups associated with arbitrary simple complex Lie algebras $\mathfrak{g}$, and we prove our first result: categories of finite-dimensional weight representations of unrolled quantum groups at odd roots of unity are relative modular, and can therefore be used to construct ETQFTs in dimension $1+1+1$.

2.1. Small quantum groups. Let $\mathfrak{g}$ be a simple complex Lie algebra of rank $n$ and dimension $2 N+n$, let $\mathfrak{h}$ be a Cartan subalgebra of $\mathfrak{g}$, let $\Phi_{+}$be a set of positive roots of $\mathfrak{g}$, and let $\mathcal{U}_{q} \mathfrak{g}$ be the associated quantum group, over a formal parameter $q$, introduced in Appendix A. Let us fix an odd integer $r \geqslant 3$ with the further condition that $r \not \equiv 0$ modulo 3 if $\mathfrak{g}=\mathfrak{g}_{2}$, and let us specialize $q$ to $e^{\frac{2 \pi i}{r}}$ in the De Concini-Kac sense [7]. Let $\bar{U}_{q} \mathfrak{g}$ denote the small quantum group of $\mathfrak{g}$, which is the $\mathbb{C}$-algebra obtained from $\mathscr{U}_{q} \mathfrak{g}$ by adding relations

$$
K_{\mu}=1, \quad E_{\alpha}^{r}=F_{\alpha}^{r}=0
$$

for every $\mu \in \Lambda_{R} \cap r \cdot \Lambda_{W}$ and every $\alpha \in \Phi_{+}$. Then $\bar{U}_{q} \mathfrak{g}$ inherits from $\mathcal{U}_{q} \mathfrak{g}$ the structure of a Hopf algebra, and we denote with $\bar{U}_{q} \mathfrak{h}$, with $\bar{U}_{q} \mathfrak{n}_{+}$, and with $\bar{U}_{q} \mathfrak{n}_{-}$ the subalgebras of $\bar{U}_{q} \mathfrak{g}$ generated by $\left\{K_{i} \mid 1 \leqslant i \leqslant n\right\}$, by $\left\{E_{i} \mid 1 \leqslant i \leqslant n\right\}$, and by $\left\{F_{i} \mid 1 \leqslant i \leqslant n\right\}$, respectively. As proved in [26], see also Theorem 30 of [17], a Poincaré-Birkhoff-Witt basis is given by

$$
\left\{\left(\prod_{k=1}^{N} F_{\beta_{k}}^{c_{k}}\right) K_{\mu}\left(\prod_{k=1}^{N} E_{\beta_{k}}^{b_{k}}\right) \begin{array}{l}
\mu \in \Lambda_{R} /\left(\Lambda_{R} \cap r \cdot \Lambda_{W}\right) \\
0 \leqslant b_{1}, \ldots, b_{N}<r \\
0 \leqslant c_{1}, \ldots, c_{N}<r
\end{array}\right\}
$$

so $\bar{U}_{q} \mathfrak{g}$ is finite-dimensional. A pivotal element is given by $K_{2 \cdot \rho} \in \bar{U}_{q} \mathfrak{g}$ where

$$
\rho:=\frac{1}{2} \cdot \sum_{k=1}^{N} \beta_{k} .
$$

Furthermore, if we consider $\bar{R}_{0} \in \bar{U}_{q} \mathfrak{h} \otimes \bar{U}_{q} \mathfrak{h}$ given by

$$
\bar{R}_{0}:=\frac{1}{\left|\Lambda_{R} /\left(\Lambda_{R} \cap r \cdot \Lambda_{W}\right)\right|} \cdot \sum_{\mu, \mu^{\prime} \in \Lambda_{R} /\left(\Lambda_{R} \cap r \cdot \Lambda_{W}\right)} q^{-\left\langle\mu, \mu^{\prime}\right\rangle} \cdot K_{\mu} \otimes K_{\mu^{\prime}}
$$

and $\bar{\Theta} \in \bar{U}_{q} \mathfrak{n}_{+} \otimes \bar{U}_{q} \mathfrak{n}_{-}$given by

$$
\bar{\Theta}:=\sum_{b_{1}, \ldots, b_{N}=0}^{r-1}\left(\prod_{k=1}^{N} \frac{\{1\}_{\beta_{k}}^{b_{k}}}{\left[b_{k}\right]_{\beta_{k}} !} q^{\frac{b_{k}\left(b_{k}-1\right)}{2}}\right) \cdot\left(\prod_{k=1}^{N} E_{\beta_{k}}^{b_{k}}\right) \otimes\left(\prod_{k=1}^{N} F_{\beta_{k}}^{b_{k}}\right)
$$

then $\bar{R}:=\bar{R}_{0} \bar{\Theta} \in \bar{U}_{q} \mathfrak{g} \otimes \bar{U}_{q} \mathfrak{g}$ is an R-matrix for $\bar{U}_{q} \mathfrak{g}$, as proved in [26], see also [25]. Next, thanks to Proposition A.5.1 of [28], a right integral $\lambda$ of $\bar{U}_{q} \mathfrak{g}$ is given by

$$
\lambda\left(\left(\prod_{k=1}^{N} F_{\beta_{k}}^{c_{k}}\right) K_{\mu}\left(\prod_{k=1}^{N} E_{\beta_{k}}^{b_{k}}\right)\right)=q^{-4\langle\rho, \rho\rangle} \delta_{\mu, 2 \cdot \rho} \prod_{k=1}^{N} \delta_{b_{k}, r-1} \prod_{k=1}^{N} \delta_{c_{k}, r-1} .
$$

This formula can be deduced from the one in [28] by remarking that Lyubashenko uses Luszitg's coproduct $\tilde{\Delta}:=(\omega \otimes \omega) \circ \Delta^{\mathrm{op}} \circ \omega$, where $\omega$ denotes the involutive algebra automorphism of $\bar{U}_{q} \mathfrak{g}$ defined by $\omega\left(E_{i}\right)=F_{i}$ and by $\omega\left(K_{i}\right)=K_{i}^{-1}$ for all integers $1 \leqslant i \leqslant n$, and by remarking that $\lambda$ is a right integral for $\Delta$ if and only if $\lambda \circ \omega$ is a left integral for $\tilde{\Delta}$. Finally, thanks to Proposition A.5.2 of [28], a two-sided cointegral $\Lambda$ of $\bar{U}_{q} \mathfrak{g}$ satisfying $\lambda(\Lambda)=1$ is given by

$$
\Lambda:=\sum_{\mu \in \Lambda_{R} /\left(\Lambda_{R} \cap r \cdot \Lambda_{W}\right)} q^{2\langle\mu, \rho\rangle} \cdot\left(\prod_{k=1}^{N} F_{\beta_{k}}^{r-1}\right) K_{\mu}\left(\prod_{k=1}^{N} E_{\beta_{k}}^{r-1}\right) .
$$


Proposition 2.1. The Hopf algebra $\bar{U}_{q} \mathfrak{g}$ is factorizable and ribbon.

This result is proved in [28], see also [25]. We denote with $\overline{\mathscr{C}}$ the ribbon category of finite-dimensional $\bar{U}_{q} \mathfrak{g}$-modules, and with $\overline{\mathrm{t}}$ the m-trace on $\operatorname{Proj}(\overline{\mathscr{C}})$ given by Theorem 1 of [1], which satisfies $\overline{\mathrm{t}}_{\bar{U}}(\Lambda \circ \varepsilon)=1$ for the regular representation $\bar{U}$ of $\bar{U}_{q} \mathfrak{g}$.

Corollary 2.2. The renormalized Hennings invariant $\mathrm{H}_{\overline{\mathscr{C}}}^{\prime}$ extends to a TQFT

$$
\mathrm{V}_{\overline{\mathscr{C}}}: \check{\mathrm{Cob}}_{\overline{\mathscr{C}}} \rightarrow \text { Vect }_{\mathbb{C}}
$$

2.2. Unrolled quantum groups. Let $U_{q}^{H} \mathfrak{g}$ denote the unrolled quantum group of $\mathfrak{g}$, which is the $\mathbb{C}$-algebra obtained from $\mathscr{U}_{q} \mathfrak{g}$ by adding generators

$$
\left\{H_{i} \mid 1 \leqslant i \leqslant n\right\}
$$

and relations

$$
\left[H_{i}, H_{j}\right]=\left[H_{i}, K_{j}\right]=0, \quad\left[H_{i}, E_{j}\right]=a_{i j} E_{j}, \quad\left[H_{i}, F_{j}\right]=-a_{i j} F_{j}, \quad E_{\alpha}^{r}=F_{\alpha}^{r}=0
$$

for every integer $1 \leqslant i, j \leqslant n$ and every positive root $\alpha \in \Phi_{+}$. Then $U_{q}^{H} \mathfrak{g}$ can be made into a pivotal Hopf algebra by setting

$$
\Delta\left(H_{i}\right)=H_{i} \otimes 1+1 \otimes H_{i}, \quad \varepsilon\left(H_{i}\right)=0, \quad S\left(H_{i}\right)=-H_{i}
$$

for every integer $1 \leqslant i \leqslant n$, and we denote with $U_{q}^{H} \mathfrak{h}$, with $U_{q}^{H} \mathfrak{n}_{+}$, and with $U_{q}^{H} \mathfrak{n}_{-}$ the subalgebras of $U_{q}^{H} \mathfrak{g}$ generated by $\left\{H_{i} \mid 1 \leqslant i \leqslant n\right\}$, by $\left\{E_{i} \mid 1 \leqslant i \leqslant n\right\}$, and by $\left\{F_{i} \mid 1 \leqslant i \leqslant n\right\}$ respectively. For every $z \in \mathbb{C}$ let us introduce the notation

$$
q^{z}:=e^{\frac{z 2 \pi i}{r}}, \quad\{z\}:=q^{z}-q^{-z} .
$$

A $U_{q}^{H} \mathfrak{g}$-module $V$ with action $\rho_{V}: U_{q}^{H} \mathfrak{g} \rightarrow \operatorname{End}_{\mathbb{k}}(V)$ is a weight module if it is a semisimple $U_{q}^{H} \mathfrak{h}$-module and if for every $\mu \in \mathfrak{h}^{*}$ and every $v \in V$ we have

$$
\rho_{V}\left(H_{i}\right)(v)=\mu\left(H_{i}\right) \cdot v \quad \forall 1 \leqslant i \leqslant n \quad \Rightarrow \quad \rho_{V}\left(K_{i}\right)(v)=q_{i}^{\mu\left(H_{i}\right)} \cdot v \quad \forall 1 \leqslant i \leqslant n,
$$

where we are identifying $\mathfrak{h}$ with the corresponding linear subspace of $U_{q}^{H} \mathfrak{h}$ in the obvious way. We denote with $\mathscr{C}^{H}$ the full subcategory of the category of finitedimensional $U_{q}^{H} \mathfrak{g}$-modules whose objects are weight modules. Then $\mathscr{C}^{H}$ can be made into a ribbon category as follows: first of all, a pivotal element is given by $K_{2 \cdot \rho}^{1-r} \in U_{q}^{H} \mathfrak{g}$, where the choice of the exponent is explained in Remark 4 of [18]. Furthermore, if $V$ and $V^{\prime}$ are objects of $\mathscr{C}^{H}$, their braiding morphism is given by

$$
\begin{array}{cccc}
c_{V, V^{\prime}}: \quad V \otimes V^{\prime} & \rightarrow & V^{\prime} \otimes V \\
v \otimes v^{\prime} & \mapsto \tau_{V, V^{\prime}}\left(R_{0, V, V^{\prime}}^{H}\left(\left(\rho_{V} \otimes \rho_{V^{\prime}}\right)\left(\Theta^{H}\right)\left(v \otimes v^{\prime}\right)\right)\right)
\end{array}
$$

for the linear maps $R_{0, V, V^{\prime}}^{H}: V \otimes V^{\prime} \rightarrow V \otimes V^{\prime}$ and $\tau_{V, V^{\prime}}: V \otimes V^{\prime} \rightarrow V^{\prime} \otimes V$ determined by

$$
R_{0, V, V^{\prime}}^{H}\left(v \otimes v^{\prime}\right):=q^{\left\langle\nu, \nu^{\prime}\right\rangle} \cdot v \otimes v^{\prime}, \quad \tau_{V, V^{\prime}}\left(v \otimes v^{\prime}\right):=v^{\prime} \otimes v
$$

for all $v \in V, v^{\prime} \in V^{\prime}$ satisfying

$$
\rho_{V}\left(H_{i}\right)(v)=\nu\left(H_{i}\right) \cdot v, \quad \rho_{V^{\prime}}\left(H_{i}\right)\left(v^{\prime}\right)=\nu^{\prime}\left(H_{i}\right) \cdot v^{\prime}
$$

for every integer $1 \leqslant i \leqslant n$, and for $\Theta^{H}=\bar{\Theta} \in U_{q}^{H} \mathfrak{n}_{+} \otimes U_{q}^{H} \mathfrak{n}_{-} \cong \bar{U}_{q} \mathfrak{n}_{+} \otimes \bar{U}_{q} \mathfrak{n}_{-}$. Thanks to Theorem 4 of [18], $\mathscr{C}^{H}$ is a ribbon category.

If we set $G:=\mathfrak{h}^{*} / \Lambda_{R}$, then $\mathscr{C}^{H}$ supports the structure of a $G$-category: indeed, for every $\gamma \in \mathfrak{h}^{*}$ we can define the homogeneous subcategory $\mathscr{C}_{[\gamma]}^{H}$ to be the full subcategory of $\mathscr{C}^{H}$ with objects given by modules whose weights are all of the form $\gamma+\mu$ for some $\mu \in \Lambda_{R}$. Furthermore, if we set $Z:=\Lambda_{R} \cap\left(r \cdot \Lambda_{W}\right)$, then we have a 
free realization $\sigma: Z \rightarrow \mathscr{C}_{[0]}^{H}$ mapping every $\kappa \in Z$ to the object $\sigma(\kappa) \in \mathscr{C}_{[0]}^{H}$ given by the vector space $\mathbb{C}$ with $U_{q}^{H} \mathfrak{g}$-action specified by

$$
\rho_{\sigma(\kappa)}\left(H_{i}\right)(1):=\kappa\left(H_{i}\right), \quad \rho_{\sigma(\kappa)}\left(E_{i}\right)(1):=0, \quad \rho_{\sigma(\kappa)}\left(F_{i}\right)(1):=0
$$

for every integer $1 \leqslant i \leqslant n$. Now the bilinear map

$$
\begin{aligned}
& \psi: G \times Z \quad \rightarrow \quad \mathbb{C}^{*} \\
& ([\gamma], \kappa) \mapsto q^{2\langle\gamma, \kappa\rangle}
\end{aligned}
$$

satisfies $c_{\sigma(\kappa), V} \circ c_{V, \sigma(\kappa)}=\psi([\gamma], \kappa) \cdot \operatorname{id}_{V \otimes \sigma(\kappa)}$ for every $\gamma \in \mathfrak{h}^{*}$, every $V \in \mathscr{C}_{[\gamma]}^{H}$, and every $\kappa \in Z$. If we consider the critical set

$$
X:=\left\{[\xi] \in \mathfrak{h}^{*} / \Lambda_{R} \mid \exists \alpha \in \Phi_{+}: 2\langle\alpha, \xi\rangle \in \mathbb{Z}\right\}
$$

then, as explained in Section 7 of [5], the category $\mathscr{C}_{[\gamma]}^{H}$ is semisimple for every $[\gamma] \in G \backslash X$. Therefore, the last relevant piece of structure we are missing is an m-trace. In order to define it, let us introduce typical $U_{q}^{H} \mathfrak{g}$-modules. First of all, we say a vector $v_{+}$of a $U_{q}^{H} \mathfrak{g}$-module $V$ is a highest weight vector if $\rho_{V}\left(E_{i}\right)\left(v_{+}\right)=0$ for every integer $1 \leqslant i \leqslant n$. Analogously, we say a vector $v_{-}$of $V$ is a lowest weight vector if $\rho_{V}\left(F_{i}\right)\left(v_{-}\right)=0$ for every integer $1 \leqslant i \leqslant n$. Then for every weight $\mu \in \mathfrak{h}^{*}$ there exists a simple finite-dimensional weight $U_{q}^{H} \mathfrak{g}$-module $V_{\mu}$ featuring a highest weight vector of weight $\mu$. This module is unique up to isomorphism, and every simple weight $U_{q}^{H} \mathfrak{g}$-module is of this form, see Proposition 33 of [17]. Every such module also has a lowest weight vector, and it is called typical if its lowest weight is given by $\mu-2(r-1) \cdot \rho$. If we consider the set

$$
\ddot{\mathfrak{h}}^{*}:=\left\{\gamma \in \mathfrak{h}^{*} \mid 2\langle\alpha, \gamma+\rho\rangle+m\langle\alpha, \alpha\rangle \notin r \mathbb{Z} \forall \alpha \in \Phi_{+}, \forall 1 \leqslant m \leqslant r-1\right\}
$$

then, thanks to Proposition 34 of [17], $V_{\gamma}$ is typical if and only if $\gamma \in \ddot{\mathfrak{h}}^{*}$. Remark that if $\gamma \in \mathfrak{h}^{*}$ satisfies $2\langle\alpha, \gamma\rangle \notin \mathbb{Z}$ for every $\alpha \in \Phi$, then $\gamma \in \ddot{\mathfrak{h}}^{*}$. This means that if $\gamma \in \mathfrak{h}^{*}$ satisfies $[\gamma] \notin X$, then $V_{\gamma}$ is typical. We also point out that, although $[(r-1) \cdot \rho] \in X$, the module $V_{(r-1) \cdot \rho}$ is always typical, because

$$
2\langle\alpha,(r-1) \cdot \rho+\rho\rangle+m\langle\alpha, \alpha\rangle=2 r\langle\alpha, \rho\rangle+m d_{\alpha}
$$

is not in $r \mathbb{Z}$ for any integer $1 \leqslant m \leqslant r-1$. Now, thanks to Lemma 7.1 of [5] and Theorem 38 of [17], every typical $U_{q}^{H} \mathfrak{g}$-module is projective and ambidextrous. Then, by combining Theorem 3.3.2 of [14] with Lemma 17 of [20], there exists a non-zero m-trace on the ideal $\operatorname{Proj}\left(\mathscr{C}^{H}\right)$ of projective objects of $\mathscr{C}^{H}$ which is unique up to scalar. Therefore, we can fix the normalization $\mathrm{d}^{H}\left(V_{(r-1) \cdot \rho}\right)=1$. Thanks to Equation (51) and Lemma 47 of [17], for every $\mu \in \ddot{\mathfrak{h}}^{*}$ we have

$$
\mathrm{d}^{H}\left(V_{\mu}\right)=\prod_{k=1}^{N} \frac{r\left\{\left\langle\mu-(r-1) \cdot \rho, \beta_{k}\right\rangle\right\}}{\left\{r\left\langle\mu-(r-1) \cdot \rho, \beta_{k}\right\rangle\right\}} .
$$

For all $\mu, \nu \in \ddot{\mathfrak{h}}^{*}$, if $f_{\mu, \nu}^{+}:=F_{\mathscr{C}_{H}}\left(T_{\mu, \nu}^{+}\right)$and $f_{\mu, \nu}^{-}:=F_{\mathscr{C}_{H}}\left(T_{\mu, \nu}^{-}\right)$for the $\mathscr{C}^{H}$-colored ribbon graphs $T_{\mu, \nu}^{+}$and $T_{\mu, \nu}^{-}$represented in Figure 3, Proposition 45 of [17] gives

$$
\mathrm{t}_{V_{\nu}}^{H}\left(f_{\mu, \nu}^{+}\right)=r^{N} q^{2\langle\mu-(r-1) \cdot \rho, \nu-(r-1) \cdot \rho\rangle}
$$

and, since $V_{\mu}^{*} \cong V_{2(r-1) \cdot \rho-\mu}$, it also gives

$$
\mathrm{t}_{V_{\nu}}^{H}\left(f_{\mu, \nu}^{-}\right)=r^{N} q^{-2\langle\mu-(r-1) \cdot \rho, \nu+(1-r) \cdot \rho\rangle}=r^{2 N} \mathrm{t}_{V_{\nu}}^{H}\left(f_{\mu, \nu}^{+}\right)^{-1} .
$$



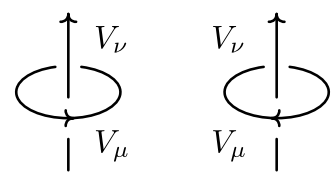

FiguRE $3 . \mathscr{C}^{H}$-colored ribbon graphs $T_{\mu, \nu}^{+}$and $T_{\mu, \nu}^{-}$.

2.3. Relative modularity. In this subsection we will prove the category $\mathscr{C}^{H}$ is relative modular, and thus yields a $Z$-graded TQFT. In order to do this, we will first need a preliminary definition. We say an endomorphism $f \in \operatorname{End}_{\mathscr{C}_{H}}(V)$ of an object $V$ of $\mathscr{C}_{[0]}^{H}$ is transparent in $\mathscr{C}_{[0]}^{H}$ if for all objects $U, W \in \mathscr{C}_{[0]}^{H}$ we have

$$
\operatorname{id}_{U} \otimes f=c_{V, U} \circ\left(f \otimes \operatorname{id}_{U}\right) \circ c_{U, V}, \quad f \otimes \operatorname{id}_{W}=c_{W, V} \circ\left(\operatorname{id}_{W} \otimes f\right) \circ c_{V, W} .
$$

Lemma 2.3. If $f \in \operatorname{End}_{\mathscr{C}^{H}}(V)$ is transparent in $\mathscr{C}_{[0]}^{H}$, then there exist some integer $m$ and some morphisms $g_{i} \in \operatorname{Hom}_{\mathscr{C} H}\left(V, \sigma\left(\kappa_{i}\right)\right)$ and $h_{i} \in \operatorname{Hom}_{\mathscr{C} H}\left(\sigma\left(\kappa_{i}\right), V\right)$ for every integer $1 \leqslant i \leqslant m$ such that

$$
f=\sum_{i=1}^{m} h_{i} \circ g_{i} .
$$

Proof. If $v_{+}$is a highest weight vector of $V_{(r-1) \cdot \rho}$ and $v$ is a weight vector of $V$ then $c_{V_{(r-1) \cdot \rho}, V}\left(v_{+} \otimes v\right)$ is proportional to $v \otimes v_{+}$because

$$
\rho_{V_{(r-1) \cdot \rho}}\left(\prod_{k=1}^{N} E_{\beta_{k}}^{b_{k}}\right)\left(v_{+}\right)=0
$$

for all integers $0 \leqslant b_{1}, \ldots, b_{N}<r$ whose sum is strictly positive. Furthermore, $c_{V, V_{(r-1) \cdot \rho}}\left(f(v) \otimes v_{+}\right)$is proportional to $v_{+} \otimes f(v)$ because $f$ is transparent in $\mathscr{C}^{H}$. But now

$$
\left\{\rho_{V_{(r-1) \cdot \rho}}\left(\prod_{k=1}^{N} F_{\beta_{k}}^{b_{k}}\right)\left(v_{+}\right) \mid 0 \leqslant b_{1}, \ldots, b_{N}<r\right\}
$$

is a basis of $V_{(r-1) \cdot \rho}$ thanks to Proposition 34 of [17]. This means that

$$
\rho_{V}\left(\prod_{k=1}^{N} E_{\beta_{k}}^{b_{k}}\right)(f(v))=0
$$

for every weight vector $v \in V$ and for all integers $0 \leqslant b_{1}, \ldots, b_{N}<r$ whose sum is strictly positive.

Analogously, if $v_{-}$is a lowest weight vector of $V_{(r-1) \cdot \rho}$ and $v$ is a weight vector of $V$ then $c_{V, V_{(r-1) \cdot \rho}}\left(v \otimes v_{-}\right)$is proportional to $v_{-} \otimes v$ because

$$
\rho_{V_{(r-1) \cdot \rho}}\left(\prod_{k=1}^{N} F_{\beta_{k}}^{b_{k}}\right)\left(v_{-}\right)=0
$$

for all integers $0 \leqslant b_{1}, \ldots, b_{N}<r$ whose sum is strictly positive. Furthermore, $c_{V_{(r-1) \cdot \rho}, V}\left(v_{-} \otimes f(v)\right)$ is proportional to $f(v) \otimes v_{-}$because $f$ is transparent in $\mathscr{C}^{H}$. But now

$$
\left\{\rho_{V_{(r-1) \cdot \rho}}\left(\prod_{k=1}^{N} E_{\beta_{k}}^{b_{k}}\right)\left(v_{-}\right) \mid 0 \leqslant b_{1}, \ldots, b_{N}<r\right\}
$$

is a basis of $V_{(r-1) \cdot \rho}$ thanks to Proposition 34 of [17]. This means that

$$
\rho_{V}\left(\prod_{k=1}^{N} F_{\beta_{k}}^{b_{k}}\right)(f(v))=0
$$


for every weight vector $v \in V$ and for all integers $0 \leqslant b_{1}, \ldots, b_{N}<r$ whose sum is strictly positive.

Now, since $K_{i}-K_{i}^{-1}=\left(q_{i}-q_{i}^{-1}\right) \cdot\left[E_{i}, F_{i}\right]$ for every integer $1 \leqslant i \leqslant n$, we get the equality $\rho_{V}\left(K_{i}\right)(f(v))-\rho_{V}\left(K_{i}^{-1}\right)(f(v))=0$ for every $v \in V$, which implies

$$
\rho_{V}\left(K_{i}\right)^{2}(f(v))=f(v) \text {. }
$$

Then, if $f(v)$ is a weight vector of weight $\kappa$, this tells us that $2\left\langle\kappa, \alpha_{i}\right\rangle \in r \mathbb{Z}$ for every integer $1 \leqslant i \leqslant n$. Since $r$ is odd and coprime with $d_{i}$ for every integer $1 \leqslant i \leqslant n$, this means precisely that $\kappa \in Z$. Therefore, each weight vector of im $f$ determines a 1-dimensional submodule which is isomorphic to $\sigma(\kappa)$ for some $\kappa \in Z$. Since im $f$ is a direct sum of its weight spaces, this means that

$$
\operatorname{im} f \cong \bigoplus_{i=1}^{m} \sigma\left(\kappa_{i}\right)
$$

for some integer $m \geqslant 1$ and some $\kappa_{1}, \ldots, \kappa_{m} \in Z$. Let $\pi_{i} \in \operatorname{Hom}_{\mathscr{C}^{H}}\left(\operatorname{im} f, \sigma\left(\kappa_{i}\right)\right)$ and $\iota_{i} \in \operatorname{Hom}_{\mathscr{C} H}\left(\sigma\left(\kappa_{i}\right), \operatorname{im} f\right)$ denote the corresponding projection and injection morphisms for every integer $1 \leqslant i \leqslant m$. We can factorize $f=\iota_{f} \circ \pi_{f}$ where $\pi_{f} \in \operatorname{Hom}_{\mathscr{C}^{H}}(V, \operatorname{im} f)$ is naturally induced by $f$ and $\iota_{f} \in \operatorname{Hom}_{\mathscr{C} H}(\operatorname{im} f, V)$ denotes inclusion. Then the result follows by setting $g_{i}:=\pi_{i} \circ \pi_{f}$ and $h_{i}:=\iota_{f} \circ \iota_{i}$ for every integer $1 \leqslant i \leqslant m$.

Let us complete $\{0\} \subset \Lambda_{R}$ to a set $\mathscr{H}_{r} \subset \Lambda_{R}$ of representatives of equivalence classes in $\Lambda_{R} / Z$. Similarly, let us choose a set $I_{G \backslash X} \subset \ddot{\mathfrak{h}}^{*}$ of representatives of equivalence classes in $G \backslash X$. If for every $\gamma \in I_{G \backslash X}$ we define $I_{[\gamma]}:=\{\gamma\}+\mathscr{H}_{r}$, then

$$
\Theta\left(\mathscr{C}_{[\gamma]}\right):=\left\{V_{\mu} \in \mathscr{C}_{[\gamma]} \mid \mu \in I_{[\gamma]}\right\}
$$

is a set of representatives of $Z$-orbits of isomorphism classes of simple objects of $\mathscr{C}_{[\gamma]}$. We have now everything in place to prove Theorem 1.3.

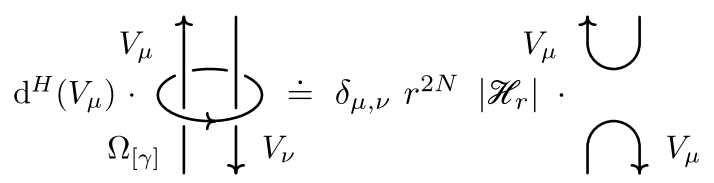

FIGURE 4. Relative modularity of $\mathscr{C}^{H}$.

Proof of Theorem 1.3. We know $\mathscr{C}^{H}$ is a non-degenerate relative pre-modular category thanks to Theorem 7.2 of [5] and to Theorem 4 of [18]. Therefore, we only need to prove that $\mathscr{C}^{H}$ satisfies the relative modularity condition of Definition 1.2. We will do this by showing the skein equivalence of Figure 4 for every $[\gamma] \in G \backslash X$, for every $\mu \in I_{G \backslash X}$, and for every $\nu \in I_{[\mu]}$, so let $f_{[\gamma], \mu, \nu}$ denote the morphism of $\mathscr{C}^{H}$ obtained by applying the Reshetikhin-Turaev functor $F_{\mathscr{C}^{H}}$ to the $\mathscr{C}^{H}$-colored ribbon graph represented in the left hand part of Figure 4, ignoring the coefficient. Thanks to the handle slide property, we have the skein equivalence of Figure 5 , which means $f_{[\gamma], \mu, \nu}$ is transparent in $\mathscr{C}_{[0]}^{H}$. Now, thanks to Lemma 2.3, we have

$$
f_{[\gamma], \mu, \nu}=\sum_{i=1}^{m} h_{[\gamma], \mu, \nu, i} \circ g_{[\gamma], \mu, \nu, i}
$$

with $g_{[\gamma], \mu, \nu, i} \in \operatorname{Hom}_{\mathscr{C}^{H}}\left(V_{\mu} \otimes V_{\nu}^{*}, \sigma\left(\kappa_{i}\right)\right)$, with $h_{[\gamma], \mu, \nu, i} \in \operatorname{Hom}_{\mathscr{C} H}\left(\sigma\left(\kappa_{i}\right), V_{\mu} \otimes V_{\nu}^{*}\right)$, and with $\kappa_{i} \in Z$ for every integer $1 \leqslant i \leqslant m$. But now $h_{[\gamma], \mu, \nu, i}=0$ unless $\mu=\nu$ and $\kappa_{i}=0$, because

$$
\operatorname{Hom}_{\mathscr{C}^{H}}\left(V_{\mu} \otimes V_{\nu}^{*}, \sigma\left(\kappa_{i}\right)\right) \cong \operatorname{Hom}_{\mathscr{C}^{H}}\left(V_{\mu}, V_{\nu} \otimes \sigma\left(\kappa_{i}\right)\right)
$$




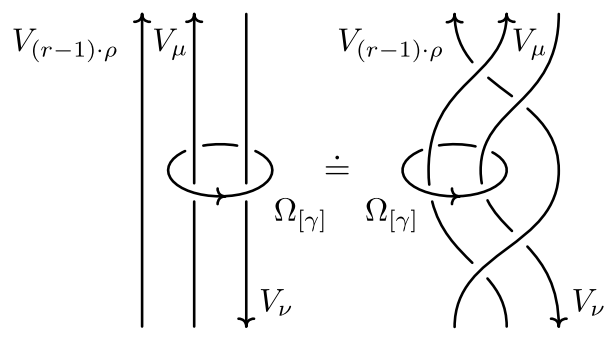

FiguRE 5. Transparency of $f_{[\gamma], \mu, \nu}$.

and because $\mathscr{H}_{r}$ is a set of representatives of equivalence classes in $\Lambda_{R} / Z$. This means that $f_{[\gamma], \mu, \mu}$ factors through the tensor unit $\mathbb{1}$. But now, since $V_{\mu}$ is simple, both $\operatorname{Hom}_{\mathscr{C}_{H}}\left(V_{\mu} \otimes V_{\mu}^{*}, \mathbb{1}\right)$ and $\operatorname{Hom}_{\mathscr{C} H}\left(\mathbb{1}, V_{\mu} \otimes V_{\mu}^{*}\right)$ are 1-dimensional. This means $f_{[\gamma], \mu, \mu}$ is a scalar multiple of $\overleftarrow{\operatorname{coev}}_{V_{\mu}} \circ \overrightarrow{\mathrm{ev}}_{V_{\mu}}$. In order to compute the proportionality coefficient let us compare the m-traces of $\overleftarrow{c o e v}_{V_{\mu}} \circ \overrightarrow{\mathrm{ev}}_{V_{\mu}}$ and of $f_{[\gamma], \mu, \mu}$. The first $\mathrm{m}$-trace is easily seen to be

$$
\mathrm{t}_{V_{\mu} \otimes V_{\mu}^{*}}^{H}\left(\overleftarrow{\operatorname{coe}}_{V_{\mu}} \circ \overrightarrow{\operatorname{ev}}_{V_{\mu}}\right)=\mathrm{t}_{V_{\mu}}^{H}\left(\mathrm{id}_{V_{\mu}}\right)=\mathrm{d}^{H}\left(V_{\mu}\right)
$$

Indeed, this follows immediately from the partial trace property of $t^{H}$. On the other hand, if

$$
f_{[\gamma], \mu, \mu}=\sum_{\nu \in\{\gamma\}+\mathscr{H}_{r}} \mathrm{~d}^{H}\left(V_{\nu}\right) \cdot f_{\nu, \mu, \mu}
$$

where $f_{\nu, \mu, \mu}$ is obtained from $f_{[\gamma], \mu, \mu}$ by replacing the label $\Omega_{[\gamma]}$ of the meridian with $V_{\nu}$, then, the second $\mathrm{m}$-trace is given by

$$
\begin{aligned}
\mathrm{t}_{V_{\mu} \otimes V_{\mu}^{*}}^{H}\left(f_{[\gamma], \mu, \mu}\right) & =\sum_{\nu \in\{\gamma\}+\mathscr{H}_{r}} \mathrm{~d}^{H}\left(V_{\nu}\right) \mathrm{t}_{V_{\mu} \otimes V_{\mu}^{*}}^{H}\left(f_{\nu, \mu, \mu}\right) \\
& =\sum_{\nu \in\{\gamma\}+\mathscr{H}_{r}} \mathrm{~d}^{H}\left(V_{\nu}\right) \mathrm{t}_{V_{\nu}}^{H}\left(f_{\mu, \nu}^{-} \circ f_{\mu, \nu}^{+}\right) \\
& =\sum_{\nu \in\{\gamma\}+\mathscr{H}_{r}} \mathrm{~d}^{H}\left(V_{\nu}\right) \mathrm{d}^{H}\left(V_{\nu}\right)^{-1} \mathrm{t}_{V_{\nu}}^{H}\left(f_{\mu, \nu}^{-}\right) \mathrm{t}_{V_{\nu}}^{H}\left(f_{\mu, \nu}^{+}\right) \\
& =r^{2 N}\left|\mathscr{H}_{r}\right|,
\end{aligned}
$$

where the morphisms $f_{\mu, \nu}^{-}$and $f_{\mu, \nu}^{+}$are represented in Figure 3. Indeed, the second equality follows from both the cyclicity and the partial trace properties of $\mathrm{t}^{H}$, using isotopy, the third equality follows from the fact that $V_{\nu}$ is simple, and the fourth equality follows from the very last equation of Subsection 2.2.

Corollary 2.4. The CGP invariant $\mathrm{N}_{\mathscr{C} H}$ extends to a Z-graded TQFT

$$
\mathbb{V}_{\mathscr{C}^{H}}^{Z}: \check{\operatorname{Cob}}_{\mathscr{C}^{H}}^{G} \rightarrow \operatorname{Vect}_{\mathbb{C}^{Z}}^{Z} \text {. }
$$

2.4. Projective generators and forgetful functor. In this subsection we prove some key technical results which will be later used for the proof Theorem 1.4. We say an object $P$ of $\overline{\mathscr{C}}$ is a projective generator of $\overline{\mathscr{C}}$ if for every object $V$ of $\operatorname{Proj}(\overline{\mathscr{C}})$ there exist some integer $m$ and some morphisms $f_{i} \in \operatorname{Hom}_{\overline{\mathscr{C}}}(V, P)$ and $g_{i} \in \operatorname{Hom}_{\overline{\mathscr{C}}}(P, V)$ for every integer $1 \leqslant i \leqslant m$ such that

$$
\operatorname{id}_{V}=\sum_{i=1}^{m} g_{i} \circ f_{i} \text {. }
$$


Remark that a natural choice for a projective generator of $\overline{\mathscr{C}}$ is the regular representation $\bar{U}$ of $\bar{U}_{q} \mathfrak{g}$. Analogously, we say an object $P$ of $\mathscr{C}_{[0]}^{H}$ is a relative projective generator of $\mathscr{C}_{[0]}^{H}$ if for every object $V$ of $\operatorname{Proj}\left(\mathscr{C}_{[0]}^{H}\right)$ there exist some integer $m$, some $\kappa_{i} \in Z$, and some morphisms $f_{i} \in \operatorname{Hom}_{\mathscr{C}^{H}}\left(V, P \otimes \sigma\left(\kappa_{i}\right)\right)$ and $g_{i} \in \operatorname{Hom}_{\mathscr{C}^{H}}\left(P \otimes \sigma\left(\kappa_{i}\right), V\right)$ for every integer $1 \leqslant i \leqslant m$ such that

$$
\operatorname{id}_{V}=\sum_{i=1}^{m} g_{i} \circ f_{i}
$$

Next, we apply the following result to $\mathscr{C}^{H}$.

Proposition 2.5. Let $\mathscr{C}$ be an abelian relative pre-modular $G$-category over an algebraically closed field $\mathbb{k}$. Then $\mathscr{C}$ has enough projectives, and every indecomposable projective object is a projective cover of a simple object.

Proof. Choose some $g \in G \backslash X$ and some simple object $V \in \mathscr{C}_{g}$. By definition $\mathscr{C}_{g}$ is semisimple, and so $V$ is projective and has epic evaluation. Then for every $W \in \mathscr{C}$ the morphism

$$
\operatorname{id}_{W} \otimes \overrightarrow{\operatorname{ev}}_{V} \in \operatorname{Hom}_{\mathscr{C}}\left(W \otimes V \otimes V^{*}, W\right) .
$$

is an epimorphism from a projective object to $W$. Thus $\mathscr{C}$ has enough projectives. Next, for the second statement, let $P$ be an indecomposable projective object of $\mathscr{C}_{h}$ for some $h \in G$. Since $X$ is small in $G$, there exists some $g \in G$ satisfying $g+h \in G \backslash X$. Then, let $V$ be a simple object of $\mathscr{C}_{g}$. The algebra $\operatorname{End}_{\mathscr{C}}(P)$ embeds into $\operatorname{End}_{\mathscr{C}}(P \otimes V) \subset \operatorname{Mat}_{N}(\mathbb{k})$ with $N$ bounded by the number of simple summands of $P \otimes V$. Then Fitting's Lemma applies to $\operatorname{End}_{\mathscr{C}}(P)$, i.e. its elements are either nilpotent or isomorphisms. We can now prove that $P$ has a unique maximal proper subobject. To see this, let us suppose by contradiction $M_{1}$ and $M_{2}$ are distinct maximal proper subobjects. Then we have an epimorphism $p \in \operatorname{Hom}_{\mathscr{C} H}\left(M_{1} \oplus M_{2}, P\right)$ which admits a section $\left(f_{1}, f_{2}\right) \in \operatorname{Hom}_{\mathscr{C} H}\left(P, M_{1} \oplus M_{2}\right)$, because $P$ is projective. If $i_{j} \in \operatorname{Hom}_{\mathscr{C}^{H}}\left(M_{j}, P\right)$ denotes the inclusion and $\tilde{f}_{j}:=i_{j} \circ f_{j}$, then $\tilde{f}_{1}+\tilde{f}_{2}=\operatorname{id}$ d $_{P}$ Since their sum is the identity of $P$, they commute, and $\tilde{f}_{1}$ and $\tilde{f}_{2}$ cannot be simultaneously nilpotent. Thanks to Fitting's Lemma, $\tilde{f}_{j}$ is an isomorphism for some $j$. This implies that $P$ is a retract of $M_{j}$, and thus $P$ is a proper subobject of $P$. Then $P \otimes V$ is a proper subobject of itself, but $P \otimes V$ is also semisimple, which is a contradiction.

We will denote by $P_{\mu}$ a projective cover of $V_{\mu}$ for any $\mu \in \mathfrak{h}^{*}$. Thanks to Proposition 2.5, if $\mathscr{H}_{r}$ denotes the set of representatives of equivalence classes in $\Lambda_{R} / Z$ of Figure 4 , then

$$
\mathbf{P}:=\bigoplus_{\mu \in \mathscr{H}_{r}} P_{\mu}
$$

is by construction a relative projective generator of $\mathscr{C}_{[0]}^{H}$.

Lemma 2.6. $\operatorname{dim}_{\mathbb{C}}\left(\operatorname{Hom}_{\mathscr{C}^{H}}(\mathbf{P}, \sigma(\kappa))\right)=\operatorname{dim}_{\mathbb{C}}\left(\operatorname{Hom}_{\mathscr{C}^{H}}(\sigma(\kappa), \mathbf{P})\right)=\delta_{0, \kappa}$ for every $\kappa \in Z$.

Proof. Since the vector space of $U_{q}^{H} \mathfrak{g}$-module morphisms from a projective indecomposable weight $U_{q}^{H} \mathfrak{g}$-module to its unique simple quotient is 1-dimensional, and since $\left\{\sigma(\kappa) \in \mathscr{C}_{[0]}^{H} \mid \kappa \in Z\right\}$ is the $Z$-orbit of the tensor unit $\mathbb{1}$, we have

$$
\operatorname{dim}_{\mathbb{C}}\left(\operatorname{Hom}_{\mathscr{C} H}(\mathbf{P}, \sigma(\kappa))\right)=\delta_{0, \kappa} .
$$

Furthermore, since the dual of an indecomposable projective weight $U_{q}^{H} \mathfrak{g}$-module is also indecomposable and projective, see for instance Proposition 6.1.3 of [11], Proposition 2.5 implies that each indecomposable projective weight $U_{q}^{H} \mathfrak{g}$-module 
$P$ has an unique simple submodule which is dual to the simple quotient of $P^{*}$. Since $\mathscr{C}^{H}$ is unimodular, see [17] and Theorem 3.1.3 of [15], $P_{0}$ is the unique indecomposable projective weight $U_{q}^{H} \mathfrak{g}$-module that constains the tensor unit $\mathbb{1}=\sigma(0)$ as a submodule, and similarly $P_{0} \otimes \sigma(\kappa)$ is the unique indecomposable projective weight $U_{q}^{H} \mathfrak{g}$-module that contains $\sigma(\kappa)$ as a submodule. Hence we have

$$
\operatorname{dim}_{\mathbb{C}}\left(\operatorname{Hom}_{\mathscr{C} H}(\sigma(\kappa), \mathbf{P})\right)=\delta_{0, \kappa} .
$$

The following result establishes a cutting property for Kirby meridians which is analogous to Lemma 3.6 of [9].

Lemma 2.7. There exist generators $\varepsilon \in \operatorname{Hom}_{\mathscr{C}^{H}}(\mathbf{P}, \mathbb{1})$ and $\boldsymbol{\Lambda} \in \operatorname{Hom}_{\mathscr{C}^{H}}(\mathbb{1}, \mathbf{P})$ satisfying $\mathrm{t}_{\mathbf{P}}^{H}(\boldsymbol{\Lambda} \circ \varepsilon)=1$ which realize the skein equivalence of Figure 6 .

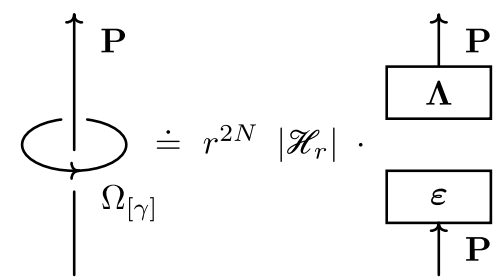

FiguRE 6. Cutting property for Kirby-colored meridians.

Proof. Thanks to Lemma 2.6, and thanks to the non-degeneracy of $\mathrm{t}^{H}$, the composition of a non-trivial morphism of $\operatorname{Hom}_{\mathscr{C} H}(\mathbf{P}, \mathbb{1})$ with a non-trivial morphism of $\operatorname{Hom}_{\mathscr{C} H}(\mathbb{1}, \mathbf{P})$ has non-zero m-trace. Therefore, let us fix a pair of generators $\varepsilon \in \operatorname{Hom}_{\mathscr{C} H}(\mathbf{P}, \mathbb{1})$ and $\boldsymbol{\Lambda} \in \operatorname{Hom}_{\mathscr{C} H}(\mathbb{1}, \mathbf{P})$ satisfying $\mathrm{t}_{\mathbf{P}}^{H}(\boldsymbol{\Lambda} \circ \varepsilon)=1$, and let us prove they realize the skein equivalence of Figure 6. If $h_{[\gamma]}$ is the morphism of $\mathscr{C}^{H}$ obtained by applying the Reshetikhin-Turaev functor $F_{\mathscr{C}_{H}}$ to the $\mathscr{C}^{H}$-colored ribbon graph represented in the left hand part of Figure 6 , the handle slide property yields the skein equivalence represented in Figure 7. This means that, thanks to Lemmas 2.3 and 2.6, the morphism $h_{[\gamma]}$ factors through the tensor unit $\mathbb{1}$. But then, since $\operatorname{Hom}_{\mathscr{C} H}(\mathbf{P}, \mathbb{1})$ and $\operatorname{Hom}_{\mathscr{C}^{H}}(\mathbb{1}, \mathbf{P})$ are both 1-dimensional, we must have $h_{[\gamma]}=\alpha \cdot \boldsymbol{\Lambda} \circ \varepsilon$ for some $\alpha \in \mathbb{C}$. Then, let us show $\alpha=r^{2 N}\left|\mathscr{H}_{r}\right|$. Proposition 2.5 implies $\operatorname{Hom}_{\mathscr{C}^{H}}\left(P_{\mu}, \mathbb{1}\right)$ is $\delta_{\mu, 0}$-dimensional for every $\mu \in \mathscr{H}_{r}$. Moreover, for every $\mu \in \ddot{\mathfrak{h}}^{*}$, the projective cover $P_{0}$ of $V_{0}=\mathbb{1}$ is a direct summand of $V_{\mu} \otimes V_{\mu}^{*}$ of multiplicity 1 . In other words, we have

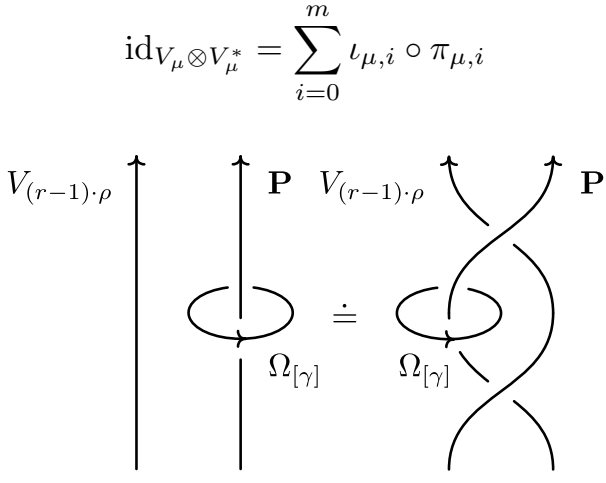

FiguRE 7 . Transparency of $h_{[\gamma]}$. 


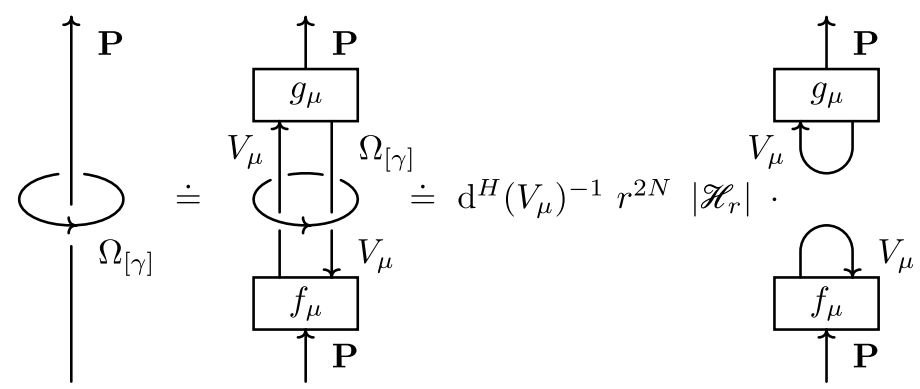

Figure 8. Cutting a P-colored edge with a Kirby-colored meridian.

for some weights $\mu_{1}, \ldots, \mu_{m} \in \Lambda_{R}$ satisfying $\mu_{0}=0$ and $\mu_{i} \neq 0$ for all integers $1 \leqslant i \leqslant m$, and for some $U_{q}^{H} \mathfrak{g}$-module morphisms $\pi_{\mu, i} \in \operatorname{Hom}_{\mathscr{C}} H\left(V_{\mu} \otimes V_{\mu}^{*}, P_{\mu_{i}}\right)$ and $\iota_{\mu, i} \in \operatorname{Hom}_{\mathscr{C} H}\left(P_{\mu_{i}}, V_{\mu} \otimes V_{\mu}^{*}\right)$. Remark that this implies

$$
\overleftarrow{\operatorname{coev}}_{V_{\mu}} \circ \overrightarrow{\mathrm{ev}}_{V_{\mu}}=\overleftarrow{\operatorname{coev}}_{V_{\mu}} \circ \overrightarrow{\mathrm{ev}}_{V_{\mu}} \circ \iota_{\mu, 0} \circ \pi_{\mu, 0}
$$

Therefore, let us fix some $\mu \in I_{G \backslash X}$, and let us consider the $U_{q}^{H} \mathfrak{g}$-module morphisms $f_{\mu}:=\iota_{\mu, 0} \circ \pi_{0} \in \operatorname{Hom}_{\mathscr{C}^{H}}\left(\mathbf{P}, V_{\mu} \otimes V_{\mu}^{*}\right)$ and $g_{\mu}:=\iota_{0} \circ \pi_{\mu, 0} \in \operatorname{Hom}_{\mathscr{C}^{H}}\left(V_{\mu} \otimes V_{\mu}^{*}, \mathbf{P}\right)$ determined by the projection $\pi_{0} \in \operatorname{Hom}_{\mathscr{C}^{H}}\left(\mathbf{P}, P_{0}\right)$ and the injection $\iota_{0} \in \operatorname{Hom}_{\mathscr{C}^{H}}\left(P_{0}, \mathbf{P}\right)$. Thanks to Figure 4, we have the skein equivalence of Figure 8. Then, let us compute the m-trace of $h_{[\gamma]}$ in two different ways. On one hand, we have

$$
\mathrm{t}_{\mathbf{P}}^{H}\left(h_{[\gamma]}\right)=\alpha \mathrm{t}_{\mathbf{P}}^{H}(\boldsymbol{\Lambda} \circ \varepsilon)=\alpha .
$$

On the other hand, we have

$$
\begin{aligned}
& \mathrm{t}_{\mathbf{P}}^{H}\left(h_{[\gamma]}\right)=\mathrm{d}^{H}\left(V_{\mu}\right)^{-1} r^{2 N}\left|\mathscr{H}_{r}\right| \mathrm{t}_{\mathbf{P}}^{H}\left(g_{\mu} \circ \overleftarrow{\operatorname{coev}}_{V_{\mu}} \circ \overrightarrow{\mathrm{ev}}_{V_{\mu}} \circ f_{\mu}\right) \\
& =\mathrm{d}^{H}\left(V_{\mu}\right)^{-1} r^{2 N}\left|\mathscr{H}_{r}\right| \mathrm{t}_{V_{\mu} \otimes V_{\mu}^{*}}^{H}\left(\overleftarrow{\operatorname{coev}}_{V_{\mu}} \circ \overrightarrow{\mathrm{eV}}_{V_{\mu}} \circ f_{\mu} \circ g_{\mu}\right) \\
& =\mathrm{d}^{H}\left(V_{\mu}\right)^{-1} r^{2 N}\left|\mathscr{H}_{r}\right| \mathrm{t}_{V_{\mu} \otimes V_{\mu}^{*}}^{H}\left(\overleftarrow{\operatorname{coev}}_{V_{\mu}} \circ \overrightarrow{\mathrm{eV}}_{V_{\mu}} \circ \iota_{\mu, 0} \circ \pi_{\mu, 0}\right) \\
& =\mathrm{d}^{H}\left(V_{\mu}\right)^{-1} r^{2 N}\left|\mathscr{H}_{r}\right| \mathrm{t}_{V_{\mu} \otimes V_{\mu}^{*}}^{H}\left(\overleftarrow{\operatorname{coev}}_{V_{\mu}} \circ \overrightarrow{\mathrm{ev}}_{V_{\mu}}\right) \\
& =\mathrm{d}^{H}\left(V_{\mu}\right)^{-1} r^{2 N}\left|\mathscr{H}_{r}\right| \mathrm{t}_{V_{\mu}}^{H}\left(\mathrm{id}_{V_{\mu}}\right) \\
& =r^{2 N}\left|\mathscr{H}_{r}\right| \text {, }
\end{aligned}
$$

where the second and fifth equalities follow from the cyclicity and the partial trace properties of $\mathrm{t}^{H}$ respectively.

Let us consider the forgetful functor $\Phi_{\mathscr{C}}: \mathscr{C}_{[0]}^{H} \rightarrow \overline{\mathscr{C}}$ which forgets the action of $H_{i}$ for all integers $1 \leqslant i \leqslant n$. If $V$ is an object of $\mathscr{C}_{[0]}^{H}$ we denote with $\bar{V}$ its image under $\Phi_{\mathscr{C}}$, and if $f$ is a morphism of $\mathscr{C}_{[0]}^{H}$ we denote with $\bar{f}$ its image under $\Phi_{\mathscr{C}}$. This induces a ribbon functor $\Phi_{\mathscr{R}}: \mathscr{R}_{\mathscr{C}_{[0]}^{H}} \rightarrow \mathscr{R}_{\overline{\mathscr{C}}}$ from the category of $\mathscr{C}_{[0]}^{H}$-colored ribbon graphs to the category of $\overline{\mathscr{C}}$-colored ribbon graphs. If $T$ is a morphism of $\mathscr{R}_{\mathscr{C} H]}$ we denote with $\bar{T}$ its image under $\Phi_{\mathscr{R}}$.

Lemma 2.8. The forgetful functor $\Phi_{\mathscr{C}}: \mathscr{C}_{[0]}^{H} \rightarrow \overline{\mathscr{C}}$ is ribbon, and it satisfies

$$
\Phi_{\mathscr{C}} \circ F_{\mathscr{C}_{[0]}^{H}}=F_{\overline{\mathscr{C}}} \circ \Phi_{\mathscr{R}} .
$$

Proof. First of all, remark that $K_{2 \cdot \rho}^{1-r}=K_{2 \cdot \rho}$ in $\bar{U}_{q} \mathfrak{g}$. Then the result follows immediately from the equality

$$
R_{0, V, V^{\prime}}^{H}=\left(\rho_{V} \otimes \rho_{V^{\prime}}\right)\left(\bar{R}_{0}\right)
$$


for all $V, V^{\prime} \in \mathscr{C}_{[0]}^{H}$, where $R_{0, V, V^{\prime}}^{H}: V \otimes V^{\prime} \rightarrow V \otimes V^{\prime}$ is defined in Subsection 2.2, and where $\bar{R}_{0} \in \bar{U}_{q} \mathfrak{h} \otimes \bar{U}_{q} \mathfrak{h}$ is defined in Subsection 2.1. To show the claim, remark

$$
\sum_{\mu^{\prime} \in \Lambda_{R} / Z} q^{\left\langle\mu, \mu^{\prime}\right\rangle}=\left|\Lambda_{R} / Z\right| \delta_{\mu, 0}
$$

for every $\mu \in \Lambda_{R}$. This means that

$$
\begin{aligned}
\left(\rho_{V} \otimes \rho_{V^{\prime}}\right)\left(\bar{R}_{0}\right)\left(v \otimes v^{\prime}\right) & =\frac{1}{\left|\Lambda_{R} / Z\right|} \cdot \sum_{\mu, \mu^{\prime} \in \Lambda_{R} / Z} q^{-\left\langle\mu, \mu^{\prime}\right\rangle} \cdot \rho_{V}\left(K_{\mu}\right)(v) \otimes \rho_{V^{\prime}}\left(K_{\mu^{\prime}}\right)\left(v^{\prime}\right) \\
& =\frac{1}{\left|\Lambda_{R} / Z\right|} \cdot \sum_{\mu, \mu^{\prime} \in \Lambda_{R} / Z} q^{\langle\mu, \nu\rangle+\left\langle\mu^{\prime}, \nu^{\prime}\right\rangle-\left\langle\mu, \mu^{\prime}\right\rangle} \cdot v \otimes v^{\prime} \\
& =\sum_{\mu \in \Lambda_{R} / Z} \delta_{\mu, \nu^{\prime}} q^{\langle\mu, \nu\rangle} \cdot v \otimes v^{\prime} \\
& =q^{\left\langle\nu, \nu^{\prime}\right\rangle} \cdot v \otimes v^{\prime}
\end{aligned}
$$

for all $v \in V, v^{\prime} \in V^{\prime}$ satisfying

$$
\rho_{V}\left(H_{i}\right)(v)=\nu\left(H_{i}\right) \cdot v, \quad \rho_{V^{\prime}}\left(H_{i}\right)\left(v^{\prime}\right)=\nu^{\prime}\left(H_{i}\right) \cdot v^{\prime}
$$

for every integer $1 \leqslant i \leqslant n$.

The forgetful functor $\Phi_{\mathscr{C}}$ preserves the property of being projective.

Lemma 2.9. If $P$ is a projective object of $\mathscr{C}_{[0]}^{H}$, then $\bar{P}$ is a projective object of $\overline{\mathscr{C}}$.

Proof. The typical $U_{q}^{H} \mathfrak{g}$-module $V_{(r-1) \cdot \rho}$ introduced in Subsection 2.2 generates $\operatorname{Proj}\left(\mathscr{C}_{[0]}^{H}\right)$ thanks to Lemma 17 of $[20]$. Then $P$ must be a direct summand of a tensor product $V_{(r-1) \cdot \rho} \otimes W$ for some $W \in \mathscr{C}_{[0]}^{H}$. Now the proof of Lemma 7.1 of [5] can be repeated to show the image $\bar{V}_{(r-1) \cdot \rho}$ of $V_{(r-1) \cdot \rho}$ under the forgetful functor $\Phi_{\mathscr{C}}$ is projective. This means $\bar{V}_{(r-1) \cdot \rho}$ generates $\operatorname{Proj}(\overline{\mathscr{C}})$, and thus $\bar{P}$, which is a direct summand of $\bar{V}_{(r-1) \cdot \rho} \otimes \bar{W}$, is projective.

In particular, the image $\overline{\mathbf{P}}$ of the relative projective generator $\mathbf{P}$ of $\mathscr{C}_{[0]}^{H}$ defined in Subsection 2.4 is projective.

Lemma 2.10. $\operatorname{dim}_{\mathbb{C}}\left(\operatorname{Hom}_{\overline{\mathscr{C}}}(\mathbb{1}, \overline{\mathbf{P}})\right)=\operatorname{dim}_{\mathbb{C}}\left(\operatorname{Hom}_{\overline{\mathscr{C}}}(\overline{\mathbf{P}}, \mathbb{1})\right)=1$.

Proof. Let $V$ be a weight $U_{q}^{H} \mathfrak{g}$-module in $\mathscr{C}_{[0]}^{H}$, and remark that its image $\bar{V}$ under $\Phi_{\mathscr{C}}$ coincides with $V$ as a vector space. Let us consider the space $\bar{V}^{\bar{U}_{q} \mathfrak{g}}$ of $\bar{U}_{q} \mathfrak{g}$ -

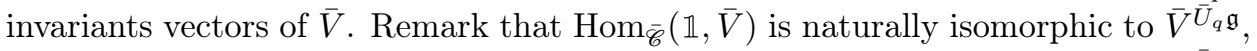
simply by identifying every morphism $f \in \operatorname{Hom}_{\overline{\mathscr{C}}}(\mathbb{1}, \bar{V})$ with the image $f(1) \in \bar{V}^{\bar{U}_{q} \mathfrak{g}}$. We claim the subspace $V^{\bar{U}_{q} \mathfrak{g}}$ of $V$ formed by vectors of $\bar{V}^{\bar{U}_{q} \mathfrak{g}}$ is a $U_{q}^{H} \mathfrak{g}$-submodule of $V$. Indeed, this follows from the commutation relations satisfied by the additional generators $H_{1}, \ldots, H_{n}$. But now remark that every weight vector of $V^{\bar{U}_{q} \mathfrak{g}}$ with respect to the action of $U_{q}^{H} \mathfrak{g}$ determines a split 1-dimensional submodule of $V^{\bar{U}_{q} \mathfrak{g}}$ which is isomorphic to $\sigma(\kappa)$ for some $\kappa \in Z$, as all 1-dimensional weight $U_{q}^{H} \mathfrak{g}$ modules in $\mathscr{C}_{[0]}^{H}$ are. This means that

$$
V^{\bar{U}_{q} \mathfrak{g}} \cong \bigoplus_{i=1}^{\operatorname{dim}_{\mathbb{C}}\left(\bar{V}^{\bar{U}_{q} \mathfrak{g}}\right)} \sigma\left(\kappa_{i}\right)
$$

with $\kappa_{i} \in Z$ for every integer $1 \leqslant i \leqslant \operatorname{dim}_{\mathbb{C}}\left(\bar{V}^{\bar{U}_{q} \mathfrak{g}}\right)$. Thus we get

$$
\operatorname{dim}_{\mathbb{C}}\left(\bar{V}^{\bar{U}_{q} \mathfrak{g}}\right) \leqslant \operatorname{dim}_{\mathbb{C}}\left(\bigoplus_{\kappa \in Z} \operatorname{Hom}_{\mathscr{C}^{H}}(\sigma(\kappa), V)\right),
$$

and the converse inequality follows from the equality $\Phi_{\mathscr{C}}(\sigma(\kappa))=\mathbb{1}$ for every $\kappa \in Z$. 
First, let us consider $V=\mathbf{P}$. Thanks to Lemma 2.6, we have

$$
\operatorname{dim}_{\mathbb{C}}\left(\bigoplus_{\kappa \in Z} \operatorname{Hom}_{\mathscr{C}^{H}}(\sigma(\kappa), \mathbf{P})\right)=1
$$

Thus, the space $\overline{\mathbf{P}}^{\bar{U}_{q} \mathfrak{g}}$ is 1-dimensional. This means

$$
\operatorname{dim}_{\mathbb{C}}\left(\operatorname{Hom}_{\overline{\mathscr{C}}}(\mathbb{1}, \overline{\mathbf{P}})\right)=1 .
$$

Next, let us consider $V=\mathbf{P}^{*}$. Thanks to Lemma 2.6, we have

$$
\operatorname{dim}_{\mathbb{C}}\left(\bigoplus_{\kappa \in Z} \operatorname{Hom}_{\mathscr{C}^{H}}\left(\sigma(\kappa), \mathbf{P}^{*}\right)\right)=\operatorname{dim}_{\mathbb{C}}\left(\bigoplus_{\kappa \in Z} \operatorname{Hom}_{\mathscr{C}^{H}}(\mathbf{P}, \sigma(-\kappa))\right)=1 .
$$

Thus, the space $\left(\overline{\mathbf{P}}^{*}\right)^{\bar{U}_{q} \mathfrak{g}}$ is 1-dimensional. This means

$$
\operatorname{dim}_{\mathbb{C}}\left(\operatorname{Hom}_{\overline{\mathscr{C}}}(\overline{\mathbf{P}}, \mathbb{1})\right)=\operatorname{dim}_{\mathbb{C}}\left(\operatorname{Hom}_{\overline{\mathscr{C}}}\left(\mathbb{1}, \overline{\mathbf{P}}^{*}\right)\right)=1 .
$$

The last result we will need requires an additional hypothesis.

Proposition 2.11. If $\operatorname{gcd}(r, \operatorname{det}(A))=1$, then $\overline{\mathbf{P}}$ is a projective generator of $\overline{\mathscr{C}}$.

Proof. Let us show that every indecomposable projective $\bar{U}_{q} \mathfrak{g}$-module $P$ is isomorphic to a direct summand of $\overline{\mathbf{P}}$. Let $V$ be the unique simple quotient of $P$. Its highest weight is a ring homomorphism $\varphi: \bar{U}_{q} \mathfrak{h} \rightarrow \mathbb{C}$ assigning to each $K_{i}$ the root of unity by which it acts on the highest weight vector of $V$. Since $r$ is coprime with $d_{1}, \ldots, d_{n}$ defined in Appendix A, there exists some $\omega \in \Lambda_{W}$ satisfying $\varphi\left(K_{i}\right)=q^{\left\langle\omega, \alpha_{i}\right\rangle}$ for every integer $1 \leqslant i \leqslant n$. Furthermore, since $r$ is coprime also with $\operatorname{det}(A)$, the matrix $\left(d_{i} a_{i j}\right)_{1 \leqslant i, j \leqslant n}$ is invertible modulo $r$. In particular, there exist $\tilde{\omega}_{i} \in \Lambda_{R}$ such that $\left\langle\tilde{\omega}_{i}, \alpha_{j}\right\rangle \equiv \delta_{i j}$ modulo $r$ for all integers $1 \leqslant i, j \leqslant n$. Then, if we set

$$
\tilde{\omega}:=\sum_{i=1}^{n}\left\langle\omega, \alpha_{i}\right\rangle \cdot \tilde{\omega}_{i} \in \Lambda_{R}
$$

the simple weight $U_{q}^{H} \mathfrak{g}$-module $V_{\tilde{\omega}} \in \mathscr{C}_{[0]}^{H}$ satisfies $\Phi_{\mathscr{C}}\left(V_{\tilde{\omega}}\right) \cong V$, because $\Phi_{\mathscr{C}}\left(V_{\tilde{\omega}}\right)$ and $V$ have the same highest weight. Moreover, if $P_{\tilde{\omega}}$ is a projective cover of $V_{\tilde{\omega}}$, then $P_{\tilde{\omega}} \otimes \sigma(\kappa)$ is isomorphic to a direct summand of $\mathbf{P}$ for some $\kappa \in Z$. But now, since $\Phi_{\mathscr{C}}\left(P_{\tilde{\omega}} \otimes \sigma(\kappa)\right)$ is projective, it must contain $P$ as a direct summand, so this proves our statement.

\section{EQUALITY OF 3-MANiFOLD INVARIANTS}

The goal for this section is to prove Theorem 1.4. We will use as a key ingredient the fact that meridians labeled with Kirby colors have the cutting property with respect to the relative projective generator $\mathbf{P}$ of $\mathscr{C}_{[0]}^{H}$, while red meridians labeled with the regular representation have the cutting property with respect to its image $\overline{\mathbf{P}}$ in $\overline{\mathscr{C}}$. The proof will require a comparison of all the ingredients that correspond to each other in the two theories. Since it is part of the hypotheses of Theorem 1.4, we will suppose throughout this section that $\operatorname{gcd}(r, \operatorname{det}(A))=1$.

3.1. Stabilized surgery presentations. In this subsection we introduce special surgery presentations of admissible decorated closed 3-manifolds which are tailored for the comparison between the CGP and the renormalized Hennings invariants. We start with a preliminary comment about our notation: we will always denote with $F_{\overline{\mathscr{C}}}$ the restriction of $F_{\lambda}$ to $\mathscr{C}$-colored blue ribbon graphs, and similarly we will always denote with $F_{\overline{\mathscr{C}}}^{\prime}$ the restriction of $F_{\lambda}^{\prime}$ to admissible closed $\overline{\mathscr{C}}$-colored blue ribbon graphs, in order to stress the absence of red edges. Next, we need to compare the m-trace $\mathrm{t}^{H}$ on $\operatorname{Proj}\left(\mathscr{C}^{H}\right)$ with the m-trace $\overline{\mathrm{t}}$ on $\operatorname{Proj}(\overline{\mathscr{C}})$. 
Remark 3.1. Both $\mathrm{t}^{H}$ and $\overline{\mathrm{t}}$ are unique up to scalar, but the chosen normalizations do not agree, as they are determined by the conditions

$$
\mathrm{t}_{\mathbf{P}}^{H}(\Lambda \circ \varepsilon)=1, \quad \overline{\mathrm{t}}_{\bar{U}}(\Lambda \circ \varepsilon)=1
$$

respectively, where $\mathbf{P}$ is the relative projective generator of $\mathscr{C}_{[0]}^{H}$ introduced in Subsection 2.4, where $\varepsilon \in \operatorname{Hom}_{\mathscr{C} H}(\mathbf{P}, \mathbb{1})$ and $\boldsymbol{\Lambda} \in \operatorname{Hom}_{\mathscr{C}^{H}}(\mathbb{1}, \mathbf{P})$ are the morphisms introduced in Lemma 2.7, where $\bar{U}$ is the regular representation of $\bar{U}_{q} \mathfrak{g}$, and where $\varepsilon$ and $\Lambda$ are the counit and the cointegral respectively. Nevertheless, $\overline{\mathrm{t}} \circ \Phi_{\mathscr{C}}$ clearly defines an m-trace on $\operatorname{Proj}\left(\mathscr{C}_{[0]}^{H}\right)$ : indeed, it obviously satisfies the cyclicity property, and the partial trace property is just a consequence of the fact that partial traces commute with the ribbon functor $\Phi_{\mathscr{C}}$ (see also Corollary 2.8 of [12] for a similar statment in the setting of finite dimensional Hopf algebras). Therefore, there exists a non-zero coefficient $\alpha \in \mathbb{C}^{*}$ such that

$$
\left.\mathrm{t}^{H}\right|_{\mathscr{C}_{[0]}^{H}}=\alpha \cdot \overline{\mathrm{t}} \circ \Phi_{\mathscr{C}}
$$

meaning that $\mathrm{t}_{V}^{H}(f)=\alpha \overline{\mathrm{t}}_{\bar{V}}(\bar{f})$ for every $V \in \operatorname{Proj}\left(\mathscr{C}_{[0]}^{H}\right)$ and every $f \in \operatorname{End}_{\mathscr{C}^{H}}(V)$.

Lemma 3.2. The renormalized invariants $F_{\mathscr{C}_{[0]}^{H}}^{\prime}$ and $F_{\overline{\mathscr{C}}}^{\prime}$ satisfy

$$
F_{\mathscr{C}_{[0]}^{H}}^{\prime}=\alpha \cdot F_{\overline{\mathscr{C}}}^{\prime} \circ \Phi_{\mathscr{R}}
$$

meaning that $F_{\mathscr{C}_{[0]}^{H}}^{\prime}(T)=\alpha F_{\overline{\mathscr{C}}}^{\prime}(\bar{T})$ for every closed admissible $\mathscr{C}_{[0]}^{H}$-colored ribbon graph $T$, where $\alpha$ is the coefficient introduced in Remark 3.1.

Proof. If a closed admissible $\mathscr{C}_{[0]}^{H}$-colored ribbon graph $T$ admits a projective edge of color $V$, and if $T_{V}$ is a cutting presentation of $T$, then we have

$$
\mathrm{t}_{V}^{H}\left(F_{\mathscr{C}_{[0]}^{H}}\left(T_{V}\right)\right)=\alpha \overline{\mathrm{t}}_{\bar{V}}\left(\Phi_{\mathscr{C}}\left(F_{\mathscr{C}_{[0]}^{H}}\left(T_{V}\right)\right)\right)=\alpha \overline{\mathrm{t}}_{\bar{V}}\left(F_{\overline{\mathscr{C}}}\left(\Phi_{\mathscr{R}}\left(T_{V}\right)\right)\right)
$$

where the second equality follows from Lemma 2.8 .

Now, let us recall the formulas defining the CGP and the renormalized Hennings invariants in the setting of Theorem 1.4. If $(M, T, 0,0)$ is a closed connected morphism of $\check{C} b_{\mathscr{C}^{H}}^{G}$, if $L=L_{1} \cup \ldots \cup L_{\ell} \subset S^{3}$ is a surgery presentation of $M$, and if we replace $(T, 0)$ with some $(\tilde{T}, \tilde{\omega})$ obtained by projective stabilization of sufficiently generic index ensuring $L$ becomes computable, as explained in Subsection 1.2 and, in greater detail, in Section 3.2 of [8], then we have

$$
\mathrm{N}_{\mathscr{C}^{H}}(M, T, 0,0)=\mathscr{D}_{\Omega}^{-1-\ell} \delta_{\Omega}^{-\sigma(L)} F_{\mathscr{C}^{H}}^{\prime}(L \cup \tilde{T}) .
$$

On the other hand, if $(M, \bar{T}, 0)$ is the closed connected morphism of Cob $_{\overline{\mathscr{C}}}$ obtained by applying the functor $\Phi_{\mathscr{R}}$ to $T \subset M$, then we have

$$
\mathrm{H}_{\overline{\mathscr{C}}}^{\prime}(M, \bar{T}, 0)=\mathscr{D}_{\lambda}^{-1-\ell} \delta_{\lambda}^{-\sigma(L)} F_{\lambda}^{\prime}(L \cup \bar{T}) .
$$

The surgery presentation $L$ is used in different ways by the two constructions. In the first case, $L$ is labeled with Kirby colors as prescribed by $\tilde{\omega}$, and thus it is not a morphism in the domain of $\Phi_{\mathscr{R}}$. In the second case, $L$ is taken to be red, and thus it is not a morphism in the image of $\Phi_{\mathscr{R}}$. In order to compare the two formulas, we introduce special morphisms of $\overline{\mathscr{C}}$ which encode these two different procedures.

First, for all weights $\mu, \nu \in \ddot{\mathfrak{h}}^{*}$ satisfying $[\mu]=[\nu] \in G \backslash X$, the tensor product $W_{\mu, \nu}:=V_{\mu} \otimes V_{\nu}^{*}$ is an object of $\mathscr{C}_{[0]}^{H}$. Therefore, since $\bar{U}$ is a projective generator of $\overline{\mathscr{C}}$, we can fix a decomposition

$$
\operatorname{id}_{\bar{W}_{\mu, \nu}}=\sum_{i=1}^{m_{\mu, \nu}} g_{\mu, \nu, i} \circ f_{\mu, \nu, i}
$$



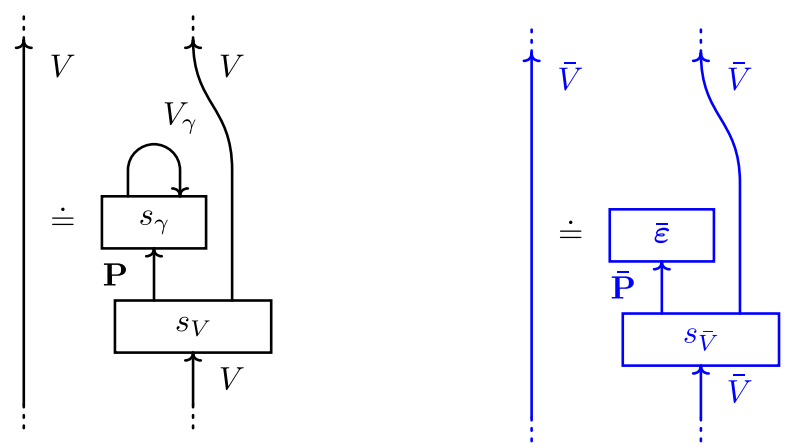

Figure 9. Projective stabilizations on $e \subset T$ and on $\bar{e} \subset \bar{T}$.

for some morphisms $f_{\mu, \nu, i} \in \operatorname{Hom}_{\overline{\mathscr{C}}}\left(\bar{W}_{\mu, \nu}, \bar{U}\right)$ and $g_{\mu, \nu, i} \in \operatorname{Hom}_{\overline{\mathscr{C}}}\left(\bar{U}, \bar{W}_{\mu, \nu}\right)$. Let us also set

$$
d_{\mu, \nu}:=\left(p_{\mu, \nu} \otimes \operatorname{id}_{W_{\mu, \nu}}\right) \circ\left(\operatorname{id}_{V_{\nu}} \otimes \overrightarrow{\operatorname{cov}}_{V_{\mu}} \otimes \operatorname{id}_{V_{\nu}^{*}}\right) \circ s_{\nu} \in \operatorname{Hom}_{\mathscr{C} H}\left(\mathbf{P}, W_{\mu, \nu}^{*} \otimes W_{\mu, \nu}\right),
$$

where $s_{\nu} \in \operatorname{Hom}_{\mathscr{C} H}\left(\mathbf{P}, W_{\nu, \nu}\right)$ is a morphism satisfying $\overrightarrow{\operatorname{ev}}_{V_{\nu}} \circ s_{\nu}=\varepsilon$, and where $p_{\mu, \nu} \in \operatorname{Hom}_{\mathscr{C} H}\left(V_{\nu} \otimes V_{\mu}^{*},\left(V_{\mu} \otimes V_{\nu}^{*}\right)^{*}\right)$ is the isomorphism coming from the pivotal structure of $\mathscr{C}^{H}$. Now, let us fix once and for all a weight $\gamma \in \ddot{\mathfrak{h}}^{*}$ satisfying $[\gamma] \in G \backslash X$. Then we denote with $h_{\Omega} \in \operatorname{Hom}_{\overline{\mathscr{G}}}\left(\overline{\mathbf{P}}, \bar{U}^{*} \otimes \bar{U}\right)$ the morphism

$$
h_{\Omega}:=\sum_{\mu \in\{\gamma\}+\mathscr{H}_{r}} \sum_{i=1}^{m_{\mu, \gamma}} \mathrm{d}^{H}\left(V_{\mu}\right) \cdot\left(\left(g_{\mu, \gamma, i}\right)^{*} \otimes f_{\mu, \gamma, i}\right) \circ \bar{d}_{\mu, \gamma} .
$$

Next, we denote with $f_{\lambda \otimes 1} \in \operatorname{Hom}_{\overline{\mathscr{C}}}\left(\bar{U}, \bar{U}^{*} \otimes \bar{U}\right)$ the unique morphism which sends the generator $1 \in \bar{U}$ to $\lambda \otimes 1 \in \bar{U}^{*} \otimes \bar{U}$, where $\lambda$ is the right integral of $\bar{U}_{q} \mathfrak{g}$, and we consider a morphism $s_{\overline{\mathbf{P}}} \in \operatorname{Hom}_{\overline{\mathscr{C}}}(\overline{\mathbf{P}}, \bar{U} \otimes \overline{\mathbf{P}})$ satisfying $\left(\varepsilon \otimes \operatorname{id}_{\overline{\mathbf{P}}}\right) \circ s_{\overline{\mathbf{P}}}=\operatorname{id}_{\overline{\mathbf{P}}}$. Then we denote with $h_{\lambda} \in \operatorname{Hom}_{\overline{\mathscr{C}}}\left(\overline{\mathbf{P}}, \bar{U}^{*} \otimes \bar{U}\right)$ the morphism

$$
h_{\lambda}:=\left(f_{\lambda \otimes 1} \otimes \bar{\varepsilon}\right) \circ s_{\overline{\mathbf{P}}}
$$

This allows us to review the recipe for the computation of the two invariants. If $(M, T, 0,0)$ is a closed morphism of $\check{C o b}_{\mathscr{C} H}^{G}$, if $e \subset T$ is a projective edge of color $V$, and if $L=L_{1} \cup \ldots \cup L_{\ell} \subset S^{3}$ is a surgery presentation of $M$, then let us fix disjoint paths $\gamma_{j} \subset S^{3} \backslash(L \cup T)$ connecting $e$ to $L_{j}$ for every integer $1 \leqslant j \leqslant \ell$. Before starting, we perform special projective stabilizations both on $e \subset T$ and on $\bar{e} \subset \bar{T}$ at the intersection point with $\gamma_{j}$ for every integer $1 \leqslant j \leqslant \ell$, as shown in Figure 9, where $s_{V} \in \operatorname{Hom}_{\mathscr{C} H}(V, \mathbf{P} \otimes V)$ is a section of $\boldsymbol{\varepsilon} \otimes \operatorname{id}_{V}$, where $s_{\bar{V}} \in \operatorname{Hom}_{\overline{\mathscr{C}}}(\bar{V}, \overline{\mathbf{P}} \otimes \bar{V})$ is its image under $\Phi_{\mathscr{C}}$, and where $s_{\gamma} \in \operatorname{Hom}_{\mathscr{C} H}\left(\mathbf{P}, W_{\gamma, \gamma}\right)$ is a morphism satisfying $\overrightarrow{\mathrm{ev}}_{V_{\gamma}} \circ s_{\gamma}=\varepsilon$. Next, we isotope the $s_{\gamma}$-colored and the $\bar{\varepsilon}$-colored coupons along the path $\gamma_{j}$ until the intersection point with $L_{j}$. This is our initial configuration.

Let us start from the CGP invariant. First, we need to slide every $V_{\gamma}$-colored edge along the corresponding component $L_{j}$, so to turn the surgery presentation $L$ into a computable one. Proposition 3.1 of [8] implies the choice of the weight $\gamma \in \ddot{\mathfrak{h}}^{*}$ satisfying $[\gamma] \in G \backslash X$ is inconsequential. This produces the $\mathscr{C}^{H}$-colored ribbon graph represented in the top-left corner of Figure 10. Up to skein equivalence of $\mathscr{C}^{H}$-colored ribbon graphs, we can replace a tubular neighborhood of $L_{j}$ as shown in the top-right corner of Figure 10, thus obtaining a $\mathscr{C}_{[0]}^{H}$-colored ribbon graph. This means we can apply the functor $\Phi_{\mathscr{R}}$ which, up to skein equivalence of $\overline{\mathscr{C}}$-colored ribbon graphs, produces the $\overline{\mathscr{C}}$-colored ribbon graph represented in the bottom-left corner of Figure 10. Again up to skein equivalence of $\overline{\mathscr{C}}$-colored ribbon graphs, we can replace a tubular neighborhood of $L_{j}$ as shown in the bottom-right corner of 


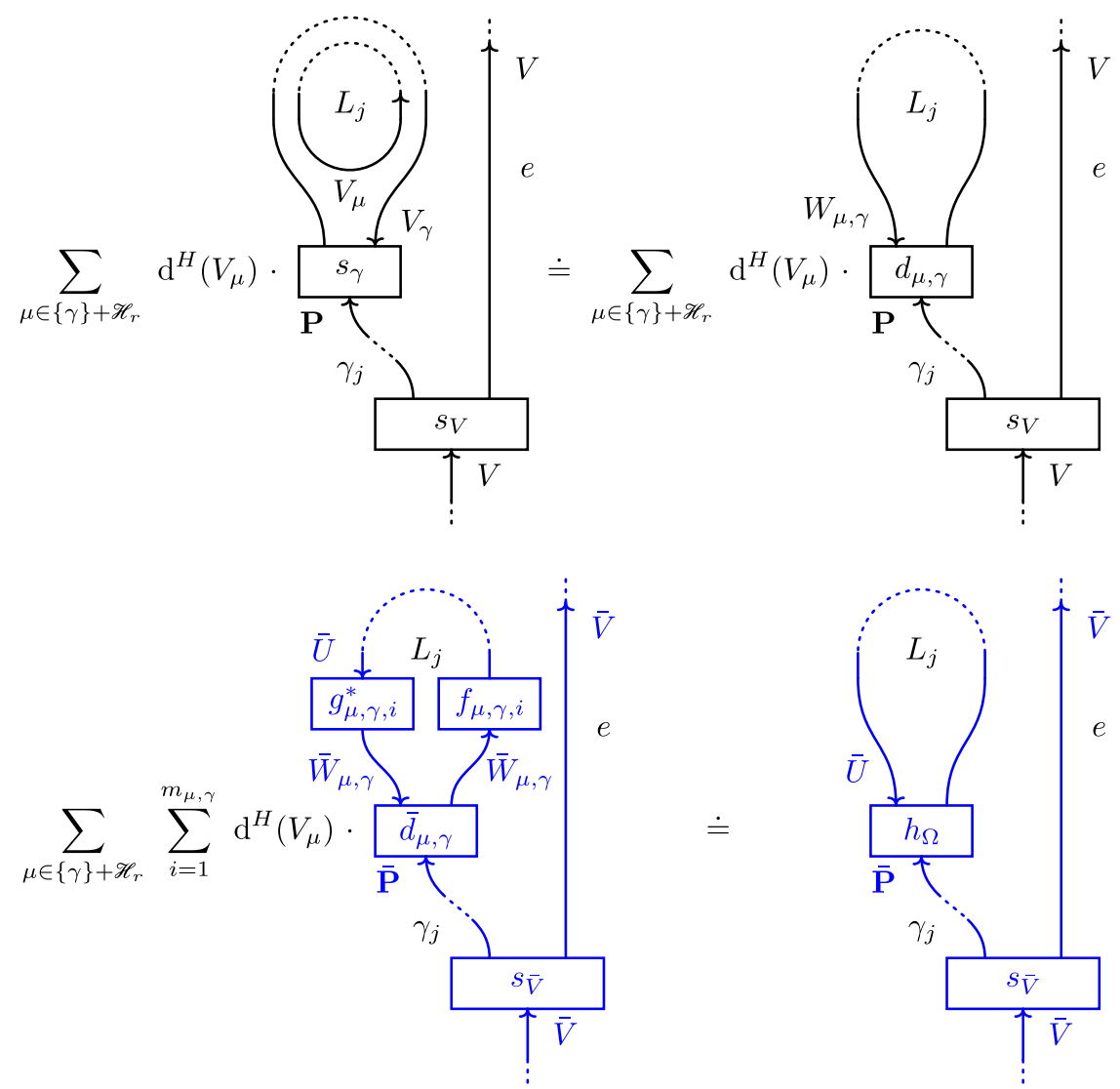

FiguRE 10. Skein equivalences of $\mathscr{C}^{H}$-colored and $\overline{\mathscr{C}}$-colored ribbon graphs defining $(L \cup \bar{T})_{h_{\Omega}}$.

Figure 10. The resulting $\overline{\mathscr{C}}$-colored ribbon graph is denoted $(L \cup \bar{T})_{h_{\Omega}}$, and is said to be obtained from the surgery presentation $L$ and from the admissible $\mathscr{C}_{[0]}^{H}$-colored ribbon graph $T$ by $\Omega$-stabilization along the paths $\gamma_{1}, \ldots, \gamma_{\ell}$. By construction, using Lemma 3.2, we have

$$
\mathrm{N}_{\mathscr{C}^{H}}(M, T, 0,0)=\alpha \mathscr{D}_{\Omega}^{-1-\ell} \delta_{\Omega}^{-\sigma(L)} F_{\mathscr{\mathscr { C }}}^{\prime}\left((L \cup \bar{T})_{h_{\Omega}}\right) .
$$

Let us move on to discuss the renormalized Hennings invariant. First, we need to interpret every component $L_{j}$ as a red edge, and to label it with the regular representation $\bar{U}$. This produces the $\overline{\mathscr{C}}$-colored bichrome graph represented in the left-hand part of Figure 11. Up to skein equivalence of $\overline{\mathscr{C}}$-colored bichrome graphs, we can turn every red component blue by replacing a tubular neighborhood of $L_{j}$ as shown in the right-hand side of Figure 11. The resulting $\overline{\mathscr{C}}$-colored ribbon graph is denoted $(L \cup \bar{T})_{h_{\lambda}}$, and is said to be obtained from the surgery presentation $L$ and from the admissible $\mathscr{C}_{[0]}^{H}$-colored ribbon graph $T$ by $\lambda$-stabilization along the paths $\gamma_{1}, \ldots, \gamma_{\ell}$. By construction, using Lemma 3.8 of [9], we have

$$
\mathrm{H}_{\mathscr{C}}^{\prime}(M, \bar{T}, 0)=\mathscr{D}_{\lambda}^{-1-\ell} \delta_{\lambda}^{-\sigma(L)} F_{\mathscr{\mathscr { C }}}^{\prime}\left((L \cup \bar{T})_{h_{\lambda}}\right) .
$$

Remark that, since $(L \cup \bar{T})_{h_{\Omega}}$ and $(L \cup \bar{T})_{h_{\lambda}}$ were obtained starting from surgery presentations computing $\mathrm{N}_{\mathscr{C}^{H}}$ and $\mathrm{H}_{\mathscr{C}_{\mathscr{C}}}^{\prime}$ through operations which do not alter the values of $F_{\mathscr{C}^{H}}^{\prime}$ and of $F_{\lambda}^{\prime}$ respectively, this means that both $F_{\mathscr{G}}^{\prime}\left((L \cup \bar{T})_{h_{\Omega}}\right)$ and $F_{\overline{\mathscr{C}}}^{\prime}\left((L \cup \bar{T})_{h_{\lambda}}\right)$ are independent of the choice of the paths $\gamma_{1}, \ldots, \gamma_{\ell}$. 


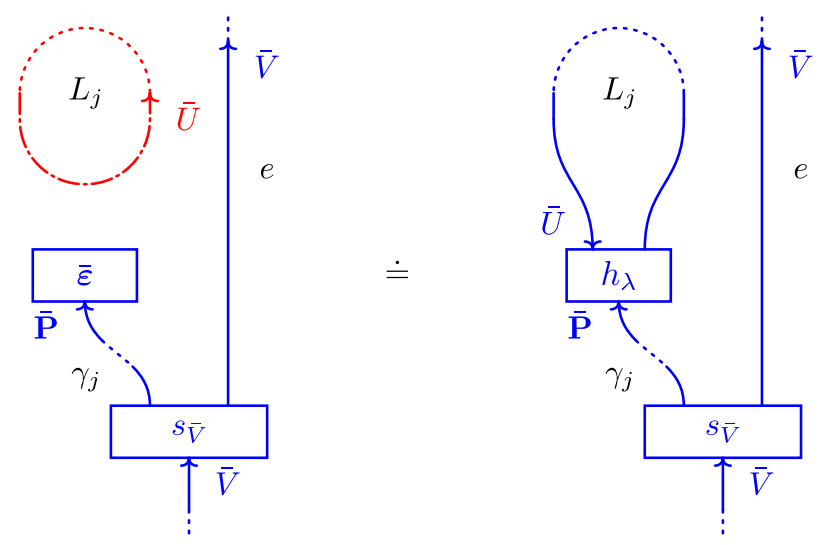

FIGURE 11. Skein equivalence of $\overline{\mathscr{C}}$-colored bichrome graphs defining $(L \cup \bar{T})_{h_{\lambda}}$.

3.2. Stabilization coefficients. Next, we need to compare $\mathscr{D}_{\Omega}$ with $\mathscr{D}_{\lambda}, \delta_{\Omega}$ with $\delta_{\lambda}$, and $h_{\Omega}$ with $h_{\lambda}$. In order to do this, we will prove a key technical result. Let $\left(\mathbb{S}^{1} \times \mathbb{S}^{1}\right)_{(-, \overline{\mathbf{P}})}$ be the object of $\check{C}_{\overline{\mathscr{C}}}$ defined by

$$
\left(\mathbb{S}^{1} \times \mathbb{S}^{1}\right)_{(-, \overline{\mathbf{P}})}:=\left(S^{1} \times S^{1}, P_{(-, \overline{\mathbf{P}})}, \mathscr{L}\right),
$$

where the blue $\overline{\mathscr{C}}$-colored ribbon set $P_{(-, \overline{\mathbf{P}})}$ is given by a single framed point with negative orientation and color $\overline{\mathbf{P}}$, and where the Lagrangian subspace $\mathscr{L}$ is generated by the homology class of the curve $\{(1,0)\} \times S^{1}$. Analogously, let $\mathbb{S}_{((-, \overline{\mathbf{P}}),(-, \overline{\mathbf{P}}),(+, \overline{\mathbf{P}}))}^{2}$ be the object of $\mathrm{Cob}_{\overline{\mathscr{C}}}$ defined by

$$
\mathbb{S}_{((-, \overline{\mathbf{P}}),(-, \overline{\mathbf{P}}),(+, \overline{\mathbf{P}}))}^{2}:=\left(S^{2}, P_{((-, \overline{\mathbf{P}}),(-, \overline{\mathbf{P}}),(+, \overline{\mathbf{P}}))},\{0\}\right),
$$

where the blue $\overline{\mathscr{C}}$-colored ribbon set $P_{((-, \overline{\mathbf{P}}),(-, \overline{\mathbf{P}}),(+, \overline{\mathbf{P}}))}$ is given by three framed points, two with negative, one with positive orientation, and all with color $\overline{\mathbf{P}}$. Let us also consider the morphism $\left(\mathbb{D}^{3} \backslash \mathbb{N}^{3}\right)_{\overline{\mathbf{P}}}:\left(\mathbb{S}^{1} \times \mathbb{S}^{1}\right)_{(-, \overline{\mathbf{P}})} \rightarrow \mathbb{S}_{((-, \overline{\mathbf{P}}),(-, \overline{\mathbf{P}}),(+, \overline{\mathbf{P}}))}^{2}$ of $\check{C}^{\circ} b_{\overline{\mathscr{C}}}$ defined by

$$
\left(\mathbb{D}^{3} \backslash \mathbb{N}^{3}\right)_{\overline{\mathbf{P}}}:=\left(D^{3} \backslash N^{3}, T_{\overline{\mathbf{P}}}, 0\right),
$$

where $N^{3} \subset D^{3}$ is an open tubular neighborhood of the curve $\{0\} \times \frac{1}{2} \cdot S^{1} \subset D^{3}$, and where the $\overline{\mathscr{C}}$-colored framed tangle $T_{\overline{\mathbf{P}}}$ is represented in Figure 12 .

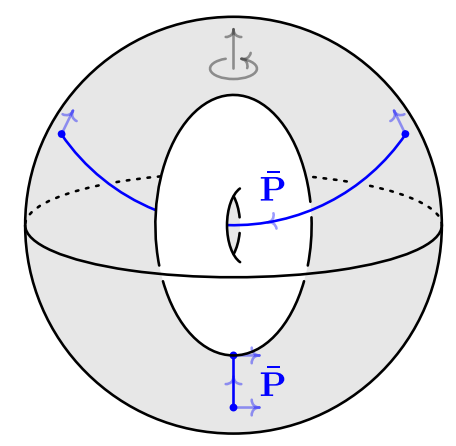

Figure 12. The morphism $\left(\mathbb{D}^{3} \backslash \mathbb{N}^{3}\right)_{\overline{\mathbf{P}}}$ of $\mathrm{Cob}_{\overline{\mathscr{G}}}$.

Lemma 3.3. The linear map

$$
\mathrm{V}_{\overline{\mathscr{C}}}\left(\left(\mathbb{D}^{3} \backslash \mathbb{N}^{3}\right)_{\overline{\mathbf{P}}}\right): \mathrm{V}_{\overline{\mathscr{C}}}\left(\left(\mathbb{S}^{1} \times \mathbb{S}^{1}\right)_{(-, \overline{\mathbf{P}})}\right) \rightarrow \mathrm{V}_{\overline{\mathscr{C}}}\left(\mathbb{S}_{((-, \overline{\mathbf{P}}),(-, \overline{\mathbf{P}}),(+, \overline{\mathbf{P}}))}^{2}\right)
$$

is injective. 


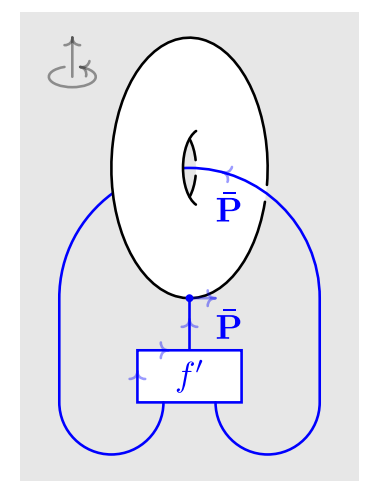

FiguRE 13. The $\overline{\mathscr{C}}$-colored ribbon graph $T_{f^{\prime}}^{\prime} \subset\left(D^{3} \backslash N^{3}\right) \cup_{S^{2}} \bar{D}^{3}$.

Proof. As we will show, the proof follows rather directly from the surjectivity of

$$
\mathrm{V}_{\overline{\mathscr{C}}}^{\prime}\left(\left(\mathbb{D}^{3} \backslash \mathbb{N}^{3}\right)_{\overline{\mathbf{P}}}\right): \mathrm{V}_{\overline{\mathscr{C}}}^{\prime}\left(\mathbb{S}_{((-, \overline{\mathbf{P}}),(-, \overline{\mathbf{P}}),(+, \overline{\mathbf{P}}))}^{2}\right) \rightarrow \mathrm{V}_{\overline{\mathscr{C}}}^{\prime}\left(\left(\mathbb{S}^{1} \times \mathbb{S}^{1}\right)_{(-, \overline{\mathbf{P}})}\right) .
$$

Indeed, a vector of the form

$$
\sum_{i=1}^{m} \alpha_{i} \cdot\left[\left(\mathbb{D}^{3} \backslash \mathbb{N}^{3}\right)_{\overline{\mathbf{P}}} \circ \mathbb{M}_{i}\right] \in \mathrm{V}_{\overline{\mathscr{C}}}\left(\mathbb{S}_{((-, \overline{\mathbf{P}}),(-, \overline{\mathbf{P}}),(+, \overline{\mathbf{P}}))}^{2}\right)
$$

for some $\alpha_{1}, \ldots, \alpha_{m} \in \mathbb{C}$ and some $\left[\mathbb{M}_{1}\right], \ldots,\left[\mathbb{M}_{m}\right] \in \mathrm{V}_{\overline{\mathscr{C}}}\left(\left(\mathbb{S}^{1} \times \mathbb{S}^{1}\right)_{(-, \overline{\mathbf{P}})}\right)$ is trivial if and only if

$$
\sum_{i=1}^{m} \alpha_{i}\left\langle\mathbb{M}^{\prime},\left(\mathbb{D}^{3} \backslash \mathbb{N}^{3}\right)_{\overline{\mathbf{P}}} \circ \mathbb{M}_{i}\right\rangle_{\mathbb{S}_{((-, \overline{\mathbf{P}}),(-, \overline{\mathbf{P}}),(+, \overline{\mathbf{P}}))}}=0
$$
for every $\left[\mathbb{M}^{\prime}\right] \in \mathrm{V}_{\overline{\mathscr{C}}}^{\prime}\left(\mathbb{S}_{((-, \overline{\mathbf{P}}),(-, \overline{\mathbf{P}}),(+, \overline{\mathbf{P}}))}^{2}\right)$, where for every object $\mathbb{\mathbb { N }}$ of $\check{C}_{\text {ob }} \overline{\mathscr{C}}_{\overline{\mathscr{C}}}$ the
linear map

$$
\langle\cdot, \cdot\rangle_{\mathbb{\Sigma}}: \mathrm{V}_{\overline{\mathscr{C}}}^{\prime}(\mathbb{\Sigma}) \otimes \mathrm{V}_{\overline{\mathscr{C}}}(\mathbb{\Sigma}) \rightarrow \mathbb{C}
$$

denotes the non-degenerate pairing induced by the universal construction in Section 3.3 of [9]. Then, since

$$
\left\langle\mathbb{M}^{\prime},\left(\mathbb{D}^{3} \backslash \mathbb{N}^{3}\right)_{\overline{\mathbf{P}}} \circ \mathbb{M}\right\rangle_{\mathbb{S}_{((-, \overline{\mathbf{P}}),(-, \overline{\mathbf{P}}),(+, \overline{\mathbf{P}}))}^{2}}=\left\langle\mathbb{M}^{\prime} \circ\left(\mathbb{D}^{3} \backslash \mathbb{N}^{3}\right)_{\overline{\mathbf{P}}}, \mathbb{M}\right\rangle_{\left(\mathbb{S}^{1} \times \mathbb{S}^{1}\right)_{(-, \overline{\mathbf{P}})}}
$$

for every $[\mathbb{M}] \in \mathrm{V}_{\overline{\mathscr{C}}}\left(\left(\mathbb{S}^{1} \times \mathbb{S}^{1}\right)_{(-, \overline{\mathbf{P}})}\right)$ and every $\left[\mathbb{M}^{\prime}\right] \in \mathrm{V}_{\overline{\mathscr{C}}}^{\prime}\left(\mathbb{S}_{((-, \overline{\mathbf{P}}),(-, \overline{\mathbf{P}}),(+, \overline{\mathbf{P}}))}^{2}\right)$, the injectivity of

$$
\mathrm{V}_{\overline{\mathscr{C}}}\left(\left(\mathbb{D}^{3} \backslash \mathbb{N}^{3}\right)_{\overline{\mathbf{P}}}\right): \mathrm{V}_{\overline{\mathscr{C}}}\left(\left(\mathbb{S}^{1} \times \mathbb{S}^{1}\right)_{(-, \overline{\mathbf{P}})}\right) \rightarrow \mathrm{V}_{\overline{\mathscr{C}}}\left(\mathbb{S}_{((-, \overline{\mathbf{P}}),(-, \overline{\mathbf{P}}),(+, \overline{\mathbf{P}}))}^{2}\right)
$$

is equivalent to the surjectivity of

$$
\mathrm{V}_{\overline{\mathscr{C}}}^{\prime}\left(\left(\mathbb{D}^{3} \backslash \mathbb{N}^{3}\right)_{\overline{\mathbf{P}}}\right): \mathrm{V}_{\overline{\mathscr{C}}}^{\prime}\left(\mathbb{S}_{((-, \overline{\mathbf{P}}),(-, \overline{\mathbf{P}}),(+, \overline{\mathbf{P}}))}^{2}\right) \rightarrow \mathrm{V}_{\overline{\mathscr{C}}}^{\prime}\left(\left(\mathbb{S}^{1} \times \mathbb{S}^{1}\right)_{(-, \overline{\mathbf{P}})}\right)
$$

In order to prove that $\mathrm{V}_{\mathscr{E}}^{\prime}\left(\left(\mathbb{D}^{3} \backslash \mathbb{N}^{3}\right)_{\overline{\mathbf{P}}}\right)$ is surjective we remark that, as soon as an object $\mathbb{\Sigma}=(\Sigma, P, \mathscr{L})$ of $\check{C} b_{\overline{\mathscr{C}}}$ features a projective blue point of $P$ in every connected component of $\Sigma$, the proof of Proposition 3.13 of [9] can be repeated to show that the linear map

$$
\begin{aligned}
& \pi_{\mathbb{\Sigma}}^{\prime}: \mathscr{V}^{\prime}\left(M^{\prime} ; \mathbb{\mathbb { Z }}\right) \quad \rightarrow \quad \mathrm{V}_{\overline{\mathscr{G}}}^{\prime}(\mathbb{\mathbb { Z }}) \\
& T^{\prime} \quad \mapsto \quad\left[M^{\prime}, T^{\prime}, 0\right]
\end{aligned}
$$

is surjective for every connected 3-dimensional cobordism $M^{\prime}$ from $\Sigma$ to $\varnothing$. This means that every vector in $\mathrm{V}_{\overline{\mathscr{C}}}^{\prime}\left(\left(\mathbb{S}^{1} \times \mathbb{S}^{1}\right)_{(-, \overline{\mathbf{P}})}\right)$ can be described by a linear combination of $\overline{\mathscr{C}}$-colored bichrome graphs inside $\left(D^{3} \backslash N^{3}\right) \cup_{S^{2}} \bar{D}^{3}$ from $P_{(-, \overline{\mathbf{P}})}$ to $\varnothing$. 
Let $T^{\prime}$ be such a $\overline{\mathscr{C}}$-colored bichrome graph. Up to isotoping its $\overline{\mathbf{P}}$-colored edge intersecting $P_{(-, \overline{\mathbf{P}})}$, we can make sure there is a projective edge of $T^{\prime}$ piercing the solid torus $N^{3}$. But now, thanks to Proposition 2.11, $\overline{\mathbf{P}}$ is a projective generator of $\overline{\mathscr{C}}$. This means that, up to skein equivalence, we can insert a pair of coupons joined by a $\overline{\mathbf{P}}$-colored edge. As a direct consequence, $T^{\prime}$ is skein equivalent to a $\overline{\mathscr{C}}$-colored ribbon graph like the one represented in Figure 13 for some $f^{\prime} \in \operatorname{Hom}_{\overline{\mathscr{C}}}\left(\overline{\mathbf{P}} \otimes \overline{\mathbf{P}}^{*}, \overline{\mathbf{P}}\right)$. Clearly every vector of this form lies in the image of $V_{\overline{\mathscr{C}}}^{\prime}\left(\left(\mathbb{D}^{3} \backslash \mathbb{N}^{3}\right)_{\overline{\mathbf{P}}}\right)$.

Let us consider now the morphisms $\left(\mathbb{S}^{1} \times \mathbb{D}^{2}\right)_{h_{\Omega}}: \varnothing \rightarrow\left(\mathbb{S}^{1} \times \mathbb{S}^{1}\right)_{(-, \overline{\mathbf{P}})}$ and $\left(\mathbb{S}^{1} \times \mathbb{D}^{2}\right)_{h_{\lambda}}: \varnothing \rightarrow\left(\mathbb{S}^{1} \times \mathbb{S}^{1}\right)_{(-, \overline{\mathbf{P}})}$ of Cob $_{\overline{\mathscr{C}}}$ defined by

$$
\begin{aligned}
\left(\mathbb{S}^{1} \times \mathbb{D}^{2}\right)_{h_{\Omega}} & :=\left(S^{1} \times D^{2}, T_{h_{\Omega}}, 0\right), \\
\left(\mathbb{S}^{1} \times \mathbb{D}^{2}\right)_{h_{\lambda}} & :=\left(S^{1} \times D^{2}, T_{h_{\lambda}}, 0\right)
\end{aligned}
$$

where the $\overline{\mathscr{C}}$-colored ribbon graphs $T_{h_{\Omega}}$ and $T_{h_{\lambda}}$ are represented in the left hand part and in the right hand part of Figure 14 respectively.

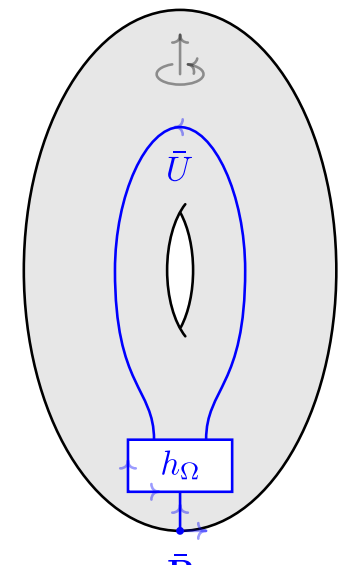

$\overline{\mathbf{P}}$

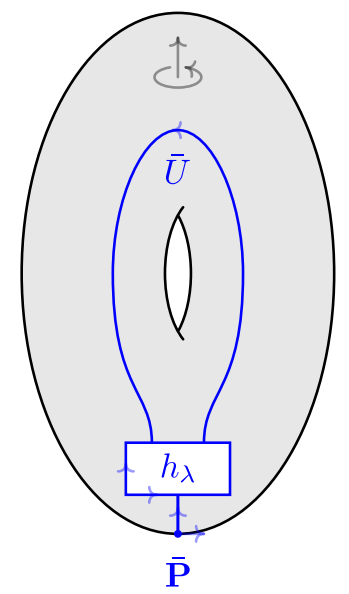

$\overline{\mathbf{P}}$

FiguRE 14. The $\overline{\mathscr{C}}$-colored ribbon graphs $T_{h_{\Omega}}, T_{h_{\lambda}} \subset S^{1} \times D^{2}$.

Lemma 3.4. The morphisms $\left(\mathbb{S}^{1} \times \mathbb{D}^{2}\right)_{h_{\Omega}}$ and $\left(\mathbb{S}^{1} \times \mathbb{D}^{2}\right)_{h_{\lambda}}$ of Čob $\check{\mathscr{C}}_{\bar{C}}$ satisfy

$$
\left[\left(\mathbb{S}^{1} \times \mathbb{D}^{2}\right)_{h_{\Omega}}\right]=\alpha \cdot\left[\left(\mathbb{S}^{1} \times \mathbb{D}^{2}\right)_{h_{\lambda}}\right] \in \mathrm{V}_{\overline{\mathscr{C}}}\left(\left(\mathbb{S}^{1} \times \mathbb{S}^{1}\right)_{(-, \overline{\mathbf{P}})}\right)
$$

where $\alpha$ is the coefficient introduced in Remark 3.1. Furthermore, there exist compatible choices for the coefficients $\mathscr{D}_{\Omega}$ and $\mathscr{D}_{\lambda}$ yielding

$$
\mathscr{D}_{\Omega}=\alpha \mathscr{D}_{\lambda}, \quad \delta_{\Omega}=\delta_{\lambda} .
$$

Proof. We start by proving $\left[\left(\mathbb{S}^{1} \times \mathbb{D}^{2}\right)_{h_{\Omega}}\right]$ and $\left[\left(\mathbb{S}^{1} \times \mathbb{D}^{2}\right)_{h_{\lambda}}\right]$ are linearly dependent in $\mathrm{V}_{\overline{\mathscr{C}}}\left(\left(\mathbb{S}^{1} \times \mathbb{S}^{1}\right)_{(-, \overline{\mathbf{P}})}\right)$. This is done by using Lemma 3.3. Indeed, on one hand, Lemma 2.7 gives the equality

$\left.\left[\left(\mathbb{D}^{3} \backslash \mathbb{N}^{3}\right)_{\overline{\mathbf{P}}}\right) \circ\left(\mathbb{S}^{1} \times \mathbb{D}^{2}\right)_{h_{\Omega}}\right]=r^{2 N}\left|\mathscr{H}_{r}\right| \cdot\left[\left(D^{3}, T_{D^{3}}, 0\right)\right] \in \mathrm{V}_{\overline{\mathscr{C}}}\left(\mathbb{S}_{((-, \overline{\mathbf{P}}),(-, \overline{\mathbf{P}}),(+, \overline{\mathbf{P}}))}^{2}\right)$,

where the $\overline{\mathscr{C}}$-colored ribbon graph $T_{D^{3}}$ is represented in Figure 15 . On the other hand, $\bar{U}$ is a projective generator of $\overline{\mathscr{C}}$, which means

$$
\operatorname{id}_{\overline{\mathbf{P}}}=\sum_{i=1}^{m} g_{\overline{\mathbf{P}}, i} \circ f_{\overline{\mathbf{P}}, i}
$$




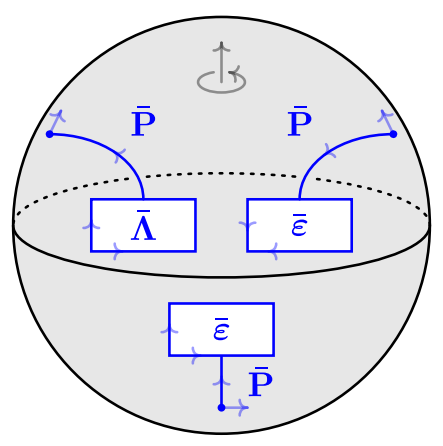

Figure 15. The $\overline{\mathscr{C}}$-colored ribbon graph $T_{D^{3}} \subset D^{3}$.

for some morphisms $f_{\overline{\mathbf{P}}, i} \in \operatorname{Hom}_{\overline{\mathscr{C}}}(\overline{\mathbf{P}}, \bar{U})$ and $g_{\overline{\mathbf{P}}, i} \in \operatorname{Hom}_{\overline{\mathscr{C}}}(\bar{U}, \overline{\mathbf{P}})$. Then, thanks to Lemma 3.6 of [9] combined with Lemma 2.10, we know there exists a non-zero coefficient $\beta \in \mathbb{C}^{*}$ giving the skein equivalence of Figure 16. This gives

$$
\left.\left[\left(\mathbb{D}^{3} \backslash \mathbb{N}^{3}\right)_{\overline{\mathbf{P}}}\right) \circ\left(\mathbb{S}^{1} \times \mathbb{D}^{2}\right)_{h_{\lambda}}\right]=\beta \cdot\left[\left(D^{3}, T_{D^{3}}, 0\right)\right] \in \mathrm{V}_{\overline{\mathscr{C}}}\left(\mathbb{S}_{((-, \overline{\mathbf{P}}),(-, \overline{\mathbf{P}}),(+, \overline{\mathbf{P}}))}^{2}\right) .
$$

Therefore, we get

$$
\beta \cdot\left[\left(\mathbb{S}^{1} \times \mathbb{D}^{2}\right)_{h_{\Omega}}\right]=r^{2 N}\left|\mathscr{H}_{r}\right| \cdot\left[\left(\mathbb{S}^{1} \times \mathbb{D}^{2}\right)_{h_{\lambda}}\right] .
$$

Next, this relation allows us to compare the stabilization coefficients. Indeed, if $T_{ \pm \Omega}$ and $T_{ \pm \lambda}$ denote the $\overline{\mathscr{C}}$-colored ribbon graphs represented in Figure 17, then, by definition of $h_{\Omega}$, we have

$$
F_{\overline{\mathscr{C}}}^{\prime}\left(T_{ \pm \Omega}\right)=\Delta_{ \pm \Omega} \overline{\mathrm{t}}_{\overline{\mathbf{P}}}(\overline{\boldsymbol{\Lambda}} \circ \overline{\boldsymbol{\varepsilon}})=\alpha \Delta_{ \pm \Omega} \mathrm{t}_{\mathbf{P}}^{H}(\boldsymbol{\Lambda} \circ \varepsilon)=\alpha \Delta_{ \pm \Omega},
$$

and analogously, by definition of $h_{\lambda}$, we have

$$
F_{\tilde{\mathscr{C}}}^{\prime}\left(T_{ \pm \lambda}\right)=\Delta_{ \pm \lambda} \overline{\mathrm{t}}_{\overline{\mathbf{P}}}(\overline{\boldsymbol{\Lambda}} \circ \overline{\boldsymbol{\varepsilon}})=\alpha \Delta_{ \pm \lambda} \mathrm{t}_{\mathbf{P}}^{H}(\boldsymbol{\Lambda} \circ \boldsymbol{\varepsilon})=\alpha \Delta_{ \pm \lambda} .
$$

This means

$$
\mathrm{H}_{\tilde{\mathscr{C}}}^{\prime}\left(S^{3}, T_{ \pm \Omega}, 0\right)=\alpha \mathscr{D}_{\lambda}^{-1} \Delta_{ \pm \Omega}, \quad \mathrm{H}_{\mathscr{\mathscr { C }}}^{\prime}\left(S^{3}, T_{ \pm \lambda}, 0\right)=\alpha \mathscr{D}_{\lambda}^{-1} \Delta_{ \pm \lambda} .
$$

But now, thanks to the previous equality, we have

$$
\beta \mathrm{H}_{\overline{\mathscr{C}}}^{\prime}\left(S^{3}, T_{ \pm \Omega}, 0\right)=r^{2 N}\left|\mathscr{H}_{r}\right| \mathrm{H}_{\mathscr{C}}^{\prime}\left(S^{3}, T_{ \pm \lambda}, 0\right) .
$$

This gives

$$
\beta \Delta_{ \pm \Omega}=r^{2 N}\left|\mathscr{H}_{r}\right| \Delta_{ \pm \lambda}
$$

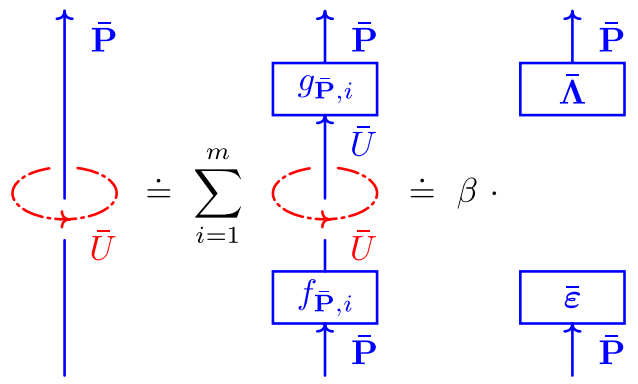

Figure 16. Cutting a $\overline{\mathbf{P}}$-colored edge with a red meridian. 

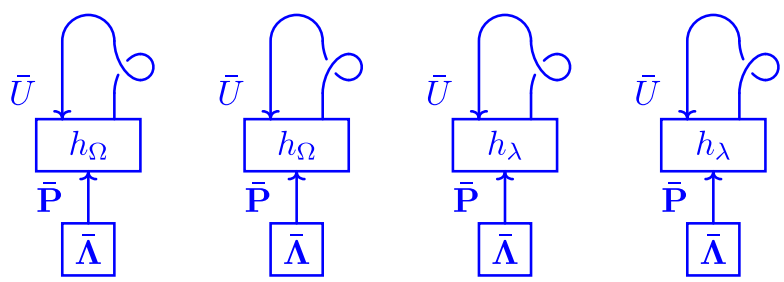

FiguRE 17. The $\overline{\mathscr{C}}$-colored ribbon graphs $T_{-\Omega}, T_{+\Omega}, T_{-\lambda}, T_{+\lambda} \subset S^{3}$.

In particular, combining this equality with the explicit value of $\Delta_{-\Omega} \Delta_{+\Omega}$ given by Figure 4, we can choose

$$
\mathscr{D}_{\Omega}=r^{N} \sqrt{\left|\mathscr{H}_{r}\right|}, \quad \mathscr{D}_{\lambda}=\frac{\beta}{r^{N} \sqrt{\left|\mathscr{H}_{r}\right|}} .
$$

This immediately implies

$$
\delta_{\Omega}=\frac{\mathscr{D}_{\Omega}}{\Delta_{-\Omega}}=\frac{\mathscr{D}_{\lambda}}{\Delta_{-\lambda}}=\delta_{\lambda}
$$

Furthermore, we can now compute the equality

$$
\beta=\frac{r^{2 N}\left|\mathscr{H}_{r}\right|}{\alpha} .
$$

Indeed, let us consider the object $\mathbb{S}_{(+, \overline{\mathbf{P}})}^{2}$ of $\mathrm{Cob}_{\overline{\mathscr{C}}}$ defined by $\left(S^{2}, P_{(+, \overline{\mathbf{P}})},\{0\}\right)$, where the blue $\overline{\mathscr{C}}$-colored ribbon set $P_{(+, \mathbf{P})}$ is given by a single framed point with positive orientation and color $\overline{\mathbf{P}}$, and let us consider the closed morphism $\mathbb{S}_{(+, \overline{\mathbf{P}})}^{2} \times \mathbb{S}^{1}$ of $\check{C}_{\overline{\mathscr{C}}}$ defined by $\left(S^{2} \times S^{1}, P_{(+, \overline{\mathbf{P}})}, 0\right)$. The strategy will be to compute the renormalized Hennings invariant of $\mathbb{S}_{(+, \overline{\mathbf{P}})}^{2} \times \mathbb{S}^{1}$ in two different ways. On one hand, the isomorphism $\mathrm{V}_{\overline{\mathscr{C}}}\left(\mathbb{S}_{(+, \overline{\mathbf{P}})}^{2}\right) \cong \operatorname{Hom}_{\overline{\mathscr{G}}}(\mathbb{\mathbb { P }}, \overline{\mathbf{P}})$ gives

$$
\mathrm{H}_{\overline{\mathscr{C}}}^{\prime}\left(\mathbb{S}_{(+, \overline{\mathbf{P}})}^{2} \times \mathbb{S}^{1}\right)=\operatorname{dim}_{\mathbb{C}}\left(\mathrm{V}_{\overline{\mathscr{C}}}\left(\mathbb{S}_{(+, \overline{\mathbf{P}})}^{2}\right)\right)=\operatorname{dim}_{\mathbb{C}}\left(\operatorname{Hom}_{\overline{\mathscr{C}}}(\mathbb{1}, \overline{\mathbf{P}})\right)=1,
$$

where the last equality follows from Lemma 2.10. On the other hand, we can choose a surgery presentation of $S^{2} \times S^{1}$ composed of a single unknot of framing 0 . This choice determines the $\overline{\mathscr{C}}$-colored bichrome graph $H_{\overline{\mathbf{P}}}$ given by a positive Hopf link of framing 0 , with one red component colored with $\bar{U}$ and one blue component colored with $\overline{\mathbf{P}}$. Therefore, we get

$$
\begin{aligned}
\mathrm{H}_{\overline{\mathscr{C}}}^{\prime}\left(\mathbb{S}_{(+, \overline{\mathbf{P}})}^{2} \times \mathbb{S}^{1}\right) & =\mathscr{D}_{\lambda}^{-2} F_{\overline{\mathscr{C}}}^{\prime}\left(H_{\overline{\mathbf{P}}}\right) \\
& =\frac{r^{2 N}\left|\mathscr{H}_{r}\right| \overline{\mathrm{t}}_{\overline{\mathbf{P}}}(\overline{\boldsymbol{\Lambda}} \circ \overline{\boldsymbol{\varepsilon}})}{\beta} \\
& =\frac{r^{2 N}\left|\mathscr{H}_{r}\right| \mathrm{t}_{\mathbf{P}}^{H}(\boldsymbol{\Lambda} \circ \varepsilon)}{\alpha \beta} \\
& =\frac{r^{2 N}\left|\mathscr{H}_{r}\right|}{\alpha \beta} .
\end{aligned}
$$

3.3. Proof of Theorem 1.4. We are now ready to prove Theorem 1.4. As explained in the proof of Lemma 3.4, using the explicit value of $\Delta_{-\Omega} \Delta_{+\Omega}$ given by Figure 4, we can choose the square roots $\mathscr{D}_{\Omega}$ and $\mathscr{D}_{\lambda}$ to be of the form

Also, let us set

$$
\mathscr{D}_{\Omega}=r^{N} \sqrt{\left|\mathscr{H}_{r}\right|}, \quad \mathscr{D}_{\lambda}=\frac{r^{N} \sqrt{\left|\mathscr{H}_{r}\right|}}{\alpha},
$$

$$
\delta:=\delta_{\Omega}=\delta_{\lambda}
$$


Proof of Theorem 1.4. If $M$ is a closed 3-manifold, and $T \subset M$ is an admissible $\mathscr{C}_{[0]}^{H}$-colored ribbon graph, then let $e \subset T$ be a projective edge of color $V$, let $L \stackrel{[0}{=} L_{1} \cup \ldots \cup L_{\ell} \subset S^{3}$ be a surgery presentation of $M$, let $\gamma_{j} \subset S^{3} \backslash(L \cup T)$ be disjoint paths connecting $e$ to $L_{j}$ for every integer $1 \leqslant j \leqslant \ell$, and let $(L \cup \bar{T})_{h_{\Omega}}$ and $(L \cup \bar{T})_{h_{\lambda}}$ be $\overline{\mathscr{C}}$-colored ribbon graphs obtained by $\Omega$-stabilization and by $\lambda$ stabilization along $\gamma_{1}, \ldots, \gamma_{\ell}$, as explained in Subsection 3.1. Then we have

$$
\begin{aligned}
\mathrm{N}_{\mathscr{C}^{H}}(M, T, 0,0) & =\alpha \mathscr{D}_{\Omega}^{-1-\ell} \delta^{-\sigma(L)} F_{\overline{\mathscr{C}}}^{\prime}\left((L \cup \bar{T})_{h_{\Omega}}\right) \\
& =\alpha^{-\ell} \mathscr{D}_{\lambda}^{-1-\ell} \delta^{-\sigma(L)} F_{\overline{\mathscr{C}}}^{\prime}\left((L \cup \bar{T})_{h_{\Omega}}\right) \\
& =\alpha^{-\ell} \mathscr{D}_{\lambda}^{-\ell} \delta^{-\sigma(L)} \mathrm{H}_{\overline{\mathscr{C}}}^{\prime}\left(S^{3},(L \cup \bar{T})_{h_{\Omega}}, 0\right) \\
& =\mathscr{D}_{\lambda}^{-\ell} \delta^{-\sigma(L)} \mathrm{H}_{\overline{\mathscr{C}}}^{\prime}\left(S^{3},(L \cup \bar{T})_{h_{\lambda}}, 0\right) \\
& =\mathscr{D}_{\lambda}^{-1-\ell} \delta^{-\sigma(L)} F_{\overline{\mathscr{C}}}^{\prime}\left((L \cup \bar{T})_{h_{\lambda}}\right) \\
& =\mathrm{H}_{\overline{\mathscr{C}}}^{\prime}(M, \bar{T}, 0)
\end{aligned}
$$

where the second and the fourth equalities follow from Lemma 3.4.

\section{Appendix A. Quantum groups}

In this appendix we collect some standard definitions related to quantum groups, see $[4,22,23,29]$ for more details. Let $\mathfrak{g}$ be a simple complex Lie algebra of rank $n$ and dimension $2 N+n$, let $B$ be its Killing form, let $\mathfrak{h}$ be a Cartan subalgebra of $\mathfrak{g}$, let $\Phi$ be the corresponding root system, let $\Phi_{+}$be a choice of a set of positive roots of $\mathfrak{g}$, and let $\left\{\alpha_{1}, \ldots, \alpha_{n}\right\}$ be an ordering of its set of simple roots. Let $A=\left(a_{i j}\right)_{1 \leqslant i, j \leqslant n}$ be the corresponding Cartan matrix, which is the integral matrix given by

$$
a_{i j}:=\frac{2 B^{*}\left(\alpha_{i}, \alpha_{j}\right)}{B^{*}\left(\alpha_{i}, \alpha_{i}\right)}
$$

where $B^{*}$ is the symmetric bilinear form on $\mathfrak{h}^{*}$ determined by the restriction of $B$ to $\mathfrak{h}$ under the isomorphism which identifies a vector $H \in \mathfrak{h}$ with the linear form $B(H, \cdot) \in \mathfrak{h}^{*}$, and let $\left\{H_{1}, \ldots, H_{n}\right\}$ be the basis of $\mathfrak{h}$ determined by $\alpha_{j}\left(H_{i}\right)=a_{i j}$ for all integers $1 \leqslant i, j \leqslant n$. For every $\alpha \in \Phi_{+}$we set

$$
d_{\alpha}:=\frac{B^{*}(\alpha, \alpha)}{\min \left\{B^{*}\left(\alpha_{i}, \alpha_{i}\right) \mid 1 \leqslant i \leqslant n\right\}}
$$

and for every integer $1 \leqslant i \leqslant n$ we use the short notation $d_{i}:=d_{\alpha_{i}}$. We denote with $\langle\cdot, \cdot\rangle$ the symmetric bilinear form on $\mathfrak{h}^{*}$ determined by $\left\langle\alpha_{i}, \alpha_{j}\right\rangle=d_{i} a_{i j}$ for all integers $1 \leqslant i, j \leqslant n$, and we denote with $\omega_{1}, \ldots, \omega_{n}$ the corresponding fundamental dominant weights, which are the vectors of $\mathfrak{h}^{*}$ determined by the condition $\left\langle\omega_{i}, \alpha_{j}\right\rangle=d_{i} \delta_{i j}$ for every $i, j=1, \ldots, n$. We denote with $\Lambda_{R}$ the root lattice, which is the subgroup of $\mathfrak{h}^{*}$ generated by simple roots, and we denote with $\Lambda_{W}$ the weight lattice, which is the subgroup of $\mathfrak{h}^{*}$ generated by fundamental dominant weights. If $q$ is a formal parameter, then for every $\alpha \in \Phi_{+}$we set $q_{\alpha}:=q^{d_{\alpha}}$, for all $k \geqslant \ell \in \mathbb{N}$ we define

$$
\begin{gathered}
\{k\}_{\alpha}:=q_{\alpha}^{k}-q_{\alpha}^{-k}, \quad[k]_{\alpha}:=\frac{\{k\}_{\alpha}}{\{1\}_{\alpha}}, \quad[k]_{\alpha} !:=[k]_{\alpha}[k-1]_{\alpha} \cdots[1]_{\alpha}, \\
{\left[\begin{array}{c}
k \\
\ell
\end{array}\right]_{\alpha}:=\frac{[k]_{\alpha} !}{[\ell]_{\alpha} ![k-\ell]_{\alpha} !},}
\end{gathered}
$$

and for every integer $1 \leqslant i \leqslant n$ we use the short notation

$$
q_{i}:=q_{\alpha_{i}}, \quad\{k\}_{i}:=\{k\}_{\alpha_{i}}, \quad[k]_{i}:=[k]_{\alpha_{i}}, \quad[k]_{i} !:=[k]_{\alpha_{i}} !, \quad\left[\begin{array}{c}
k \\
\ell
\end{array}\right]_{i}:=\left[\begin{array}{c}
k \\
\ell
\end{array}\right]_{\alpha_{i}} .
$$


Let $\mathcal{U}_{q} \mathfrak{g}$ denote the quantum group of $\mathfrak{g}$, which is the $\mathbb{C}(q)$-algebra with generators

$$
\left\{K_{i}, K_{i}^{-1}, E_{i}, F_{i} \mid 1 \leqslant i \leqslant n\right\}
$$

and relations

$$
\begin{gathered}
K_{i} K_{i}^{-1}=K_{i}^{-1} K_{i}=1, \quad\left[K_{i}, K_{j}\right]=0, \\
K_{i} E_{j} K_{i}^{-1}=q_{i}^{a_{i j}} \cdot E_{j}, \quad K_{i} F_{j} K_{i}^{-1}=q_{i}^{-a_{i j}} \cdot F_{j}, \\
{\left[E_{i}, F_{j}\right]=\delta_{i j} \cdot \frac{K_{i}-K_{i}^{-1}}{q_{i}-q_{i}^{-1}}}
\end{gathered}
$$

for all integers $1 \leqslant i, j \leqslant n$ and

$$
\begin{aligned}
& \sum_{k=0}^{1-a_{i j}}(-1)^{k}\left[\begin{array}{c}
1-a_{i j} \\
k
\end{array}\right]_{i} \cdot E_{i}^{k} E_{j} E_{i}^{1-a_{i j}-k}=0, \\
& \sum_{k=0}^{1-a_{i j}}(-1)^{k}\left[\begin{array}{c}
1-a_{i j} \\
k
\end{array}\right]_{i} \cdot F_{i}^{k} F_{j} F_{i}^{1-a_{i j}-k}=0
\end{aligned}
$$

for all integers $1 \leqslant i, j \leqslant n$ with $i \neq j$. Then $\mathcal{U}_{q} \mathfrak{g}$ can be made into a Hopf algebra by setting

$$
\begin{array}{lll}
\Delta\left(K_{i}\right)=K_{i} \otimes K_{i}, & \varepsilon\left(K_{i}\right)=1, & S\left(K_{i}\right)=K_{i}^{-1}, \\
\Delta\left(E_{i}\right)=E_{i} \otimes K_{i}+1 \otimes E_{i}, & \varepsilon\left(E_{i}\right)=0, & S\left(E_{i}\right)=-E_{i} K_{i}^{-1}, \\
\Delta\left(F_{i}\right)=F_{i} \otimes 1+K_{i}^{-1} \otimes F_{i}, & \varepsilon\left(F_{i}\right)=0, & S\left(F_{i}\right)=-K_{i} F_{i}
\end{array}
$$

for all integers $1 \leqslant i \leqslant n$. For every

$$
\mu=\sum_{i=1}^{n} m_{i} \cdot \alpha_{i} \in \Lambda_{R}
$$

we use the notation

$$
K_{\mu}:=\prod_{i=1}^{n} K_{i}^{m_{i}},
$$

and for every $\alpha \in \Phi_{+}$we define root vectors $E_{\alpha}$ and $F_{\alpha}$ as follows: first, we consider the Weyl group $W$ of $\mathfrak{g}$ associated with $\mathfrak{h}$, which is the subgroup of $\operatorname{GL}\left(\mathfrak{h}^{*}\right)$ generated by reflections

$$
\begin{array}{cccc}
s_{i}: & \mathfrak{h}^{*} & \rightarrow & \mathfrak{h}^{*} \\
& \alpha_{j} & \mapsto & \alpha_{j}-a_{i j} \cdot \alpha_{i}
\end{array}
$$

for every integer $1 \leqslant i \leqslant n$. Next, we consider the unique element $w_{0} \in W$ corresponding to a word of maximal length in the generators. The choice of a decomposition $w_{0}=s_{i_{1}} \circ \cdots \circ s_{i_{N}}$ determines a total order on the set of positive roots

$$
\Phi_{+}=\left\{\alpha_{i_{1}}, s_{i_{1}}\left(\alpha_{i_{2}}\right), \ldots,\left(s_{i_{1}} \circ \cdots \circ s_{i_{N-1}}\right)\left(\alpha_{i_{N}}\right)\right\} .
$$


Then, for every integer $1 \leqslant i \leqslant n$, we consider the automorphism $T_{i}$ of $\mathscr{U}_{q} \mathfrak{g}$ determined by

$$
\begin{aligned}
T_{i}\left(K_{j}\right) & :=K_{j} K_{i}^{-a_{i j}}, \\
T_{i}\left(E_{j}\right) & := \begin{cases}-F_{i} K_{i} & i=j, \\
\sum_{k=0}^{-a_{i j}}(-1)^{k} \frac{q_{i}^{a_{i j}+k}}{[k]_{i} !\left[-a_{i j}-k\right]_{i} !} \cdot E_{i}^{k} E_{j} E_{i}^{-a_{i j}-k} & i \neq j,\end{cases} \\
T_{i}\left(F_{j}\right) & := \begin{cases}-K_{i}^{-1} E_{i} & i=j, \\
\sum_{k=0}^{-a_{i j}}(-1)^{-a_{i j}-k} \frac{q_{i}^{k}}{[k]_{i} !\left[-a_{i j}-k\right]_{i} !} \cdot F_{i}^{k} F_{j} F_{i}^{-a_{i j}-k} & i \neq j .\end{cases}
\end{aligned}
$$

Now, for every integer $1 \leqslant k \leqslant N$, we set

$$
\beta_{k}:=\left(s_{i_{1}} \circ \cdots \circ s_{i_{k-1}}\right)\left(\alpha_{i_{k}}\right) \in \Phi_{+}
$$

and

$$
E_{\beta_{k}}:=\left(T_{i_{1}} \circ \cdots \circ T_{i_{k-1}}\right)\left(E_{i_{k}}\right) \in \mathscr{U}_{q} \mathfrak{g}, \quad F_{\beta_{k}}:=\left(T_{i_{1}} \circ \cdots \circ T_{i_{k-1}}\right)\left(F_{i_{k}}\right) \in \mathscr{U}_{q} \mathfrak{g} .
$$

\section{REFERENCES}

[1] A. Beliakova, C. Blanchet, A. Gainutdinov, Modified Trace is a Symmetrised Integral, arXiv:1801.00321 [math.QA]

[2] C. Blanchet, F. Costantino, N. Geer, B. Patureau-Mirand, Non-Semisimple TQFTs, Reidemeister Torsion and Kashaev's Invariants, Advances in Mathematics, Volume 301, 1 October 2016, Pages 1-78

[3] C. Blanchet, N. Habegger, G. Masbaum, P. Vogel, Topological Quantum Field Theories Derived from the Kauffman Bracket, Topology, Volume 34, Issue 4, 1995, Pages 883-927

[4] V. Chari, A. Pressley, A Guide to Quantum Groups, Cambridge University Press, July 1995

[5] F. Costantino, N. Geer, B. Patureau-Mirand, Quantum Invariants of 3-Manifolds via Link Surgery Presentations and Non-Semi-Simple Categories, Journal of Topology, Volume 7, Number 4, 2014, Pages 1005-1053

[6] T. Creutzig, A. Gainutdinov, I. Runkel, A Quasi-Hopf Algebra for the Triplet Vertex Operator Algebra, Communications in Contemporary Mathematics, 2019

[7] C. De Concini, V. Kac, Representations of Quantum Groups at Roots of 1, Operator Algebras, Unitary Representations, Enveloping Algebras, and Invariant Theory: Actes du Colloque en l'Honneur de Jacques Dixmier, Progress in Mathematics, Volume 92, Birkhäuser Boston, Pages 471-506, 1990

[8] M. De Renzi, Non-Semisimple Extended Topological Quantum Field Theories, arXiv: 1703.07573 [math.GT]

[9] M. De Renzi, N. Geer, B. Patureau, Renormalized Hennings Invariants and 2+1-TQFTs, Communications in Mathematical Physics, Volume 362, Issue 3, September 2018, Pages 855907

[10] M. De Renzi, A. Gainutdinov, N. Geer, B. Patureau, I. Runkel, 3-Dimensional TQFTs from Non-Semisimple Modular Categories, In Preparation

[11] P. Etingof, S. Gelaki, D. Nikshych, V. Ostrik, Tensor Categories, Mathematical Surveys and Monographs, Volume 205, American Mathematical Society, 2015

[12] A. Fontalvo Orozco, A. Gainutdinov, Module Traces and Hopf Group-Coalgebras, arXiv: 1809.01122 [math.QA]

[13] A. Gainutdinov, S. Lentner, T. Ohrmann, Modularization of Small Quantum Groups, arXiv:1809.02116 [math.QA]

[14] N. Geer, J. Kujawa, B. Patureau-Mirand, Generalized Trace and Modified Dimension Functions on Ribbon Categories, Selecta Mathematica, Volume 17, Issue 2, June 2011, Pages 453-504

[15] N. Geer, J. Kujawa, B. Patureau-Mirand, Ambidextrous Objects and Trace Functions for Nonsemisimple Categories, Proceedings of the American Mathematical Society, Volume 141, 2013, Pages 2963-2978

[16] N. Geer, J. Kujawa, B. Patureau-Mirand, M-Traces in (Non-Unimodular) Pivotal Categories, arXiv:1809.00499 [math.RT]

[17] N. Geer, B. Patureau-Mirand, Topological Invariants from Non-Restricted Quantum Groups, Algebraic \& Geometric Topology, Volume 13, Number 6, 2013, Pages 3305-3363 
[18] N. Geer, B. Patureau-Mirand, The Trace on Projective Representations of Quantum Groups, Letters in Mathematical Physics, January 2018, Volume 108, Issue 1, Pages 117-140

[19] N. Geer, B. Patureau-Mirand, V. Turaev, Modified Quantum Dimensions and Re-Normalized Link Invariants, Compositio Mathematica, Volume 145, Issue 1, January 2009, Pages 196-212

[20] N. Geer, B. Patureau-Mirand, A. Virelizier, Traces on Ideals in Pivotal Categories, Quantum Topology, Volume 4, Issue 1, 2013, Pages 91-124

[21] M. Hennings, Invariants of Links and 3-Manifolds Obtained from Hopf Algebras, Journal of the London Mathematical Society, Volume 54, Issue 3, 1996, Pages 594-624.

[22] J. Jantzen, Lectures on Quantum Groups, Graduate Studies in Mathematics, Volume 6, American Mathematical Society, Providence, RI, 1996

[23] A. Klimyk, K. Schmüdgen, Quantum Groups and Their Representations, Springer-Verlag Berlin Heidelberg, 1997

[24] S. Lentner, D. Nett, New R-Matrices for Small Quantum Groups, Algebras and Representation Theory, Volume 18, Issue 6, December 2015, Pages 1649-1673

[25] S. Lentner, T. Ohrmann, Factorizable R-Matrices for Small Quantum Groups, SIGMA, Volume 13, Number 076, 2017, Pages 1-25

[26] G. Lusztig, Quantum Groups at Roots of 1, Geometriae Dedicata, Volume 35, Issue 1-3, September 1990, Pages 89-113

[27] G. Lusztig, Introduction to Quantum Groups, Progress in Mathematics, Volume 110, Birkhauser, Boston, 1993

[28] V. Lyubashenko, Invariants of 3-Manifolds and Projective Representations of Mapping Class Groups via Quantum Groups at Roots of Unity, Communications in Mathematical Physics, Volume 172, Issue 3, September 1995, Pages 467-516

[29] S. Majid, Foundations of Quantum Group Theory, Cambridge University Press, 2011

[30] C. Negron, Log-Modular Quantum Groups at Even Roots of Unity and the Quantum Frobenius I, arXiv:1812.02277 [math.QA]

[31] D. Radford, Hopf Algebras, Series on Knots and Everything, Volume 49, World Scientific, December 2011

[32] V. Turaev, Quantum Invariants of Knots and 3-Manifolds, Berlin, Boston: De Gruyter, 1994

Department of Mathematics, Faculty of Science and Engineering, Waseda UniverSity, 3-4-1 Ökubo, Shinjuku-ku, Tokyo, 169-8555, Japan

E-mail address: m.derenzi@kurenai.waseda.jp

Mathematics \& Statistics, Utah State University, Logan, Utah 84322, USA

E-mail address: nathan.geer@gmail.com

Univ. Bretagne - Sud, UMR 6205, LMBA, F-56000 Vannes, France

E-mail address: bertrand.patureau@univ-ubs.fr 